\title{
SOBOLEV AND TRUDINGER TYPE INEQUALITIES ON GRAND MUSIELAK-ORLICZ-MORREY SPACES
}

\author{
Fumi-Yuki Maeda, Yoshihiro Mizuta, Takao Ohno and Tetsu Shimomura
}

\author{
4-24 Furue-higashi-machi, Nishi-ku, Hiroshima 733-0872, Japan; fymaeda@h6.dion.ne.jp \\ Hiroshima Institute of Technology, Department of Mechanical Systems Engineering \\ 2-1-1 Miyake Saeki-ku Hiroshima 731-5193, Japan; y.mizuta.5x@it-hiroshima.ac.jp \\ Oita University, Faculty of Education and Welfare Science \\ Dannoharu Oita-city 870-1192, Japan; t-ohno@oita-u.ac.jp \\ Hiroshima University, Graduate School of Education, Department of Mathematics \\ Higashi-Hiroshima 739-8524, Japan; tshimo@hiroshima-u.ac.jp
}

\begin{abstract}
Our aim in this paper is to establish generalizations of Sobolev's inequality and Trudinger's inequality for general potentials of functions in grand Musielak-Orlicz-Morrey spaces.
\end{abstract}

\section{Introduction}

Grand Lebesgue spaces were introduced in [15] for the study of Jacobian. They play important roles also in the theory of partial differential equations (see [10], [16] and [29], etc.). The generalized grand Lebesgue spaces appeared in [12], where the existence and uniqueness of the non-homogeneous $N$-harmonic equations were studied. The boundedness of the maximal operator on the grand Lebesgue spaces was studied in [9]. For variable exponent Lebesgue spaces, see [6] and [7]. In [21] and [17], grand Morrey spaces and generalized grand Morrey spaces were introduced. For Morrey spaces, we refer to [24] and [27]. Further, grand Morrey spaces of variable exponent were considered in [11].

On the other hand, the classical Sobolev's inequality for Riesz potentials of $L^{p_{-}}$ functions (see, e.g. [2, Theorem 3.1.4 (b)]) has been extended to various function spaces. For Morrey spaces, Sobolev's inequality was studied in [1], [27], [5], [25], etc., for Morrey spaces of variable exponent in [3], [13], [14], [22], [23], etc., for grand Morrey spaces in [21] and [17], and also for grand Morrey spaces of variable exponent in [11]. Recently, Sobolev's inequality has been extended by the authors [19] to an inequality for general potentials of functions in Musielak-Orlicz-Morrey spaces.

The classical Trudinger's inequality for Riesz potentials of $L^{p}$-functions (see, e.g. [2, Theorem 3.1.4 (c)]) has been also extended to function spaces as above; see [22], [23] for Morrey spaces of variable exponent, [11] for grand Morrey spaces of variable exponent and [20] for Musielak-Orlicz-Morrey spaces.

In this paper, we define (generalized) grand Musielak-Orlicz-Morrey space on a bounded open set in $\mathbf{R}^{N}$ and give a Sobolev type inequality as well as a Trudinger type inequality for general potentials of functions in such spaces.

doi:10.5186/aasfm.2015.4027

2010 Mathematics Subject Classification: Primary 31B15, 46E35.

Key words: Riesz potentials, maximal functions, Sobolev's inequality, Trudinger's inequality, grand Musielak-Orlicz-Morrey spaces. 
Throughout this paper, let $C$ denote various constants independent of the variables in question. The symbols $g \lesssim h$ and $g \sim h$ means that $g \leq C h$ and $C^{-1} h \leq g \leq C h$ for some constant $C>0$ respectively.

\section{Preliminaries}

Let $G$ be a bounded open set in $\mathbf{R}^{N}$ and let $d_{G}$ denote the diameter of $G$. We consider a function

$$
\Phi(x, t)=t \phi(x, t): G \times[0, \infty) \rightarrow[0, \infty)
$$

satisfying the following conditions $(\Phi 1)-(\Phi 4)$ :

$(\Phi 1) \phi(\cdot, t)$ is measurable on $G$ for each $t \geq 0$ and $\phi(x, \cdot)$ is continuous on $[0, \infty)$ for each $x \in G$;

(\$2) there exists a constant $A_{1} \geq 1$ such that

$$
A_{1}^{-1} \leq \phi(x, 1) \leq A_{1} \quad \text { for all } x \in G ;
$$

(Ф3) there exists a constant $\varepsilon_{0}>0$ such that $t \mapsto t^{-\varepsilon_{0}} \phi(x, t)$ is uniformly almost increasing, namely there exists a constant $A_{2} \geq 1$ such that

$$
t^{-\varepsilon_{0}} \phi(x, t) \leq A_{2} s^{-\varepsilon_{0}} \phi(x, s)
$$

for all $x \in G$ whenever $0<t<s$;

(Ф4) there exists a constant $A_{3} \geq 1$ such that

$$
\phi(x, 2 t) \leq A_{3} \phi(x, t) \quad \text { for all } x \in G \text { and } t>0 .
$$

Note that (\$3) implies that

$$
t^{-\varepsilon} \phi(x, t) \leq A_{2} s^{-\varepsilon} \phi(x, s)
$$

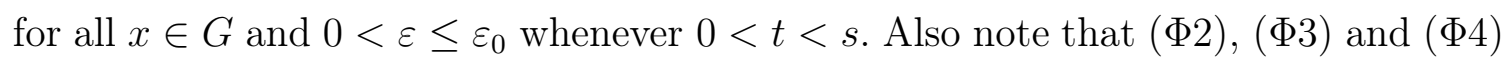
imply

$$
0<\inf _{x \in G} \phi(x, t) \leq \sup _{x \in G} \phi(x, t)<\infty
$$

for each $t>0$ and there exists $\omega>1$ such that

$$
\left(A_{1} A_{2}\right)^{-1} t^{1+\varepsilon_{0}} \leq \Phi(x, t) \leq A_{1} A_{2} A_{3} t^{\omega}
$$

for $t \geq 1$; in fact we can take $\omega \geq 1+\log A_{3} / \log 2$.

We shall also consider the following condition:

(Ф5) for every $\gamma>0$, there exists a constant $B_{\gamma} \geq 1$ such that

$$
\phi(x, t) \leq B_{\gamma} \phi(y, t)
$$

whenever $|x-y| \leq \gamma t^{-1 / N}$ and $t \geq 1$.

Let $\bar{\phi}(x, t)=\sup _{0 \leq s \leq t} \phi(x, s)$ and

$$
\bar{\Phi}(x, t)=\int_{0}^{t} \bar{\phi}(x, r) d r .
$$

Then $\bar{\Phi}(x, \cdot)$ is convex and

$$
\frac{1}{2 A_{3}} \Phi(x, t) \leq \bar{\Phi}(x, t) \leq A_{2} \Phi(x, t)
$$

for all $x \in G$ and $t \geq 0$. 
Example 2.1. Let $p(\cdot)$ and $q_{j}(\cdot), j=1, \ldots, k$, be measurable functions on $G$ such that

$$
1<p^{-}:=\inf _{x \in G} p(x) \leq \sup _{x \in G} p(x)=: p^{+}<\infty
$$

and

$$
-\infty<q_{j}^{-}:=\inf _{x \in G} q_{j}(x) \leq \sup _{x \in G} q_{j}(x)=: q_{j}^{+}<\infty, \quad j=1, \ldots k .
$$

Set $L(t):=\log (e+t), L^{(1)}(t)=L(t)$ and $L^{(j)}(t)=L\left(L^{(j-1)}(t)\right), j=2, \ldots$. Then,

$$
\Phi_{p(\cdot),\left\{q_{j}(\cdot)\right\}}(x, t)=t^{p(x)} \prod_{j=1}^{k}\left(L^{(j)}(t)\right)^{q_{j}(x)}
$$

satisfies $(\Phi 1),(\Phi 2),(\Phi 3)$ with $0<\varepsilon_{0}<p^{-}-1$ and $(\Phi 4)$. (2.1) holds for any $\omega>p^{+}$. $\Phi_{p(\cdot),\left\{q_{j}(\cdot)\right\}}(x, t)$ satisfies $(\Phi 5)$ if $p(\cdot)$ is log-Hölder continuous, namely

$$
|p(x)-p(y)| \leq \frac{C_{p}}{L(1 /|x-y|)} \quad(x \neq y)
$$

and $q_{j}(\cdot)$ is $(j+1)$-log-Hölder continuous, namely

$$
\left|q_{j}(x)-q_{j}(y)\right| \leq \frac{C_{q_{j}}}{L^{(j+1)}(1 /|x-y|)} \quad(x \neq y)
$$

for $j=1, \ldots, k$ (cf. [19, Example 2.1]).

We also consider a function $\kappa(x, r): G \times\left(0, d_{G}\right) \rightarrow(0, \infty)$ satisfying the following conditions:

$(\kappa 1) \kappa(x, \cdot)$ is continuous on $\left(0, d_{G}\right)$ for each $x \in G$ and satisfies the uniform doubling condition: there is a constant $Q_{1} \geq 1$ such that

$$
Q_{1}^{-1} \kappa(x, r) \leq \kappa\left(x, r^{\prime}\right) \leq Q_{1} \kappa(x, r)
$$

for all $x \in G$ whenever $0<r \leq r^{\prime} \leq 2 r<d_{G}$;

$(\kappa 2) r \mapsto r^{-\delta} \kappa(x, r)$ is uniformly almost increasing for some $\delta>0$, namely there is a constant $Q_{2}>0$ such that

$$
r^{-\delta} \kappa(x, r) \leq Q_{2} s^{-\delta} \kappa(x, s)
$$

for all $x \in G$ whenever $0<r<s<d_{G}$;

$(\kappa 3)$ there is a constant $Q_{3} \geq 1$ such that

$$
Q_{3}^{-1} \min \left(1, r^{N}\right) \leq \kappa(x, r) \leq Q_{3}
$$

for all $x \in G$ and $0<r<d_{G}$.

Example 2.2. Let $\nu(\cdot)$ and $\beta(\cdot)$ be functions on $G$ such that $\nu^{-}:=\inf _{x \in G} \nu(x)>$ $0, \nu^{+}:=\sup _{x \in G} \nu(x) \leq N$ and $-c(N-\nu(x)) \leq \beta(x) \leq c$ for all $x \in G$ and some constant $c>0$. Then $\kappa(x, r)=r^{\nu(x)}(\log (e+1 / r))^{\beta(x)}$ satisfies $(\kappa 1),(\kappa 2)$ and $(\kappa 3)$; we can take any $0<\delta<\nu^{-}$for $(\kappa 2)$. by

Given $\Phi(x, t)$ and $\kappa(x, r)$, we define the Musielak-Orlicz-Morrey space $L^{\Phi, \kappa}(G)$

$$
L^{\Phi, \kappa}(G)=\left\{f \in L_{l o c}^{1}(G) ; \sup _{x \in G, 0<r<d_{G}} \frac{\kappa(x, r)}{|B(x, r)|} \int_{B(x, r) \cap G} \Phi(y,|f(y)|) d y<\infty\right\} .
$$


It is a Banach space with respect to the norm

$$
\|f\|_{\Phi, \kappa ; G}=\inf \left\{\lambda>0 ; \sup _{x \in G, 0<r<d_{G}} \frac{\kappa(x, r)}{|B(x, r)|} \int_{B(x, r) \cap G} \bar{\Phi}(y,|f(y)| / \lambda) d y \leq 1\right\}
$$

(cf. [26]).

In case $\kappa(x, r)=r^{N}, L^{\Phi, \kappa}(G)$ is the Musielak-Orlicz space

$$
L^{\Phi}(G)=\left\{f \in L_{\mathrm{loc}}^{1}(G) ; \int_{G} \Phi(y,|f(y)|) d y<\infty\right\}
$$

with the norm

$$
\|f\|_{\Phi ; G}=\inf \left\{\lambda>0 ; \int_{G} \Phi(y,|f(y)| / \lambda) d y \leq 1\right\} .
$$

Remark 2.3. The Musielak-Orlicz spaces $L^{\Phi}(G)$ include

- Orlicz spaces defined by Young functions satisfying the doubling condition;

- variable exponent Lebesgue spaces.

The Musielak-Orlicz-Morrey spaces $L^{\Phi, \kappa}(G)$ include Morrey spaces as well as variable exponent Morrey spaces.

\section{Grand Musielak-Orlicz-Morrey space}

For $\varepsilon \geq 0$, set $\Phi_{\varepsilon}(x, t):=t^{-\varepsilon} \Phi(x, t)=t^{1-\varepsilon} \phi(x, t)$. Then, $\Phi_{\varepsilon}(x, t)$ satisfies $(\Phi 1)$, ( $\Phi 2)$ with the same $A_{1}$ and $(\Phi 4)$ with the same $A_{3}$. If $\Phi(x, t)$ satisfies $(\Phi 5)$, then so does $\Phi_{\varepsilon}(x, t)$ with the same $\left\{B_{\gamma}\right\}_{\gamma>0}$.

If $0 \leq \varepsilon<\varepsilon_{0}$, then $\Phi_{\varepsilon}(x, t)$ satisfies $(\Phi 3)$ with $\varepsilon_{0}$ replaced by $\varepsilon_{0}-\varepsilon$ and the same $A_{2}$. It follows that

$$
\frac{1}{2 A_{3}} \Phi_{\varepsilon}(x, t) \leq \overline{\Phi_{\varepsilon}}(x, t) \leq A_{2} \Phi_{\varepsilon}(x, t)
$$

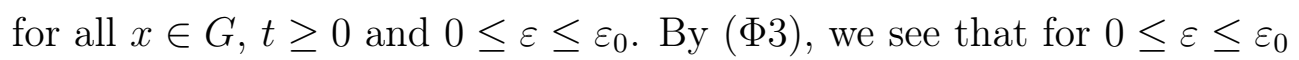

$$
\Phi_{\varepsilon}(x, a t) \begin{cases}\leq A_{2} a \Phi_{\varepsilon}(x, t) & \text { if } 0 \leq a \leq 1, \\ \geq A_{2}^{-1} a \Phi_{\varepsilon}(x, t) & \text { if } a \geq 1\end{cases}
$$

Let

$$
\tilde{\sigma}=\sup \left\{\sigma \geq 0: r^{N-\sigma} \kappa(x, r)^{-1} \text { is bounded on } G \times\left(0, \min \left(1, d_{G}\right)\right)\right\} .
$$

By $(\kappa 2), 0 \leq \tilde{\sigma} \leq N$. If $\tilde{\sigma}=0$, then let $\sigma_{0}=0$; otherwise fix any $\sigma_{0} \in(0, \tilde{\sigma})$. We also take $\delta_{0}$ such that $0<\delta_{0}<\delta$ for $\delta$ in $(\kappa 2)$.

For $-\delta_{0} \leq \sigma \leq \sigma_{0}$, set

$$
\kappa_{\sigma}(x, r)=r^{\sigma} \kappa(x, r)
$$

for $x \in G$ and $0<r<d_{G}$. Then $\kappa_{\sigma}(x, r)$ satisfies $(\kappa 1),(\kappa 2)$ and $(\kappa 3)$ with constants independent of $\sigma$.

Lemma 3.1. For $0 \leq \varepsilon \leq \varepsilon_{0}$, let

$$
\Phi_{\varepsilon}^{-1}(x, s)=\sup \left\{t>0: \Phi_{\varepsilon}(x, t)<s\right\} \quad(x \in G, s>0) .
$$

Then there exists $r_{0} \in\left(0, \min \left(1, d_{G}\right)\right)$ such that $\kappa_{\sigma}(x, r) \leq 1$ and

$$
\left.\Phi_{\varepsilon}^{-1}\left(x, \kappa_{\sigma}(x, r)^{-1}\right)\right) \geq 1
$$

for all $x \in G, 0<r \leq r_{0},-\delta_{0} \leq \sigma \leq \sigma_{0}$ and $0<\varepsilon \leq \varepsilon_{0}$. 
Proof. By $(\kappa 2)$ and $(\kappa 3)$,

$$
\kappa_{\sigma}(x, r) \leq Q_{2} Q_{3} \min \left(1, d_{G}\right)^{-\delta} r^{\delta+\sigma} \leq Q_{2} Q_{3} \min \left(1, d_{G}\right)^{-\delta} r^{\delta-\delta_{0}}
$$

for $x \in G, 0<r<\min \left(1, d_{G}\right)$ and $-\delta_{0} \leq \sigma \leq \sigma_{0}$. Hence, there is $r^{\prime} \in\left(0, \min \left(1, d_{G}\right)\right)$ such that $\kappa_{\sigma}(x, r) \leq 1$ for $x \in G, 0<r \leq r^{\prime}$ and $-\delta_{0} \leq \sigma \leq \sigma_{0}$. By (2.1), we see that

$$
\Phi_{\varepsilon}^{-1}\left(x, \kappa_{\sigma}(x, r)^{-1}\right) \geq C^{-1} \kappa_{\sigma}(x, r)^{-1 / \omega} \geq C^{\prime-1} r^{-\left(\delta-\delta_{0}\right) / \omega}
$$

whenever $x \in G, 0<r \leq r^{\prime},-\delta_{0} \leq \sigma \leq \sigma_{0}$ and $0<\varepsilon \leq \varepsilon_{0}$ with constants $C, C^{\prime}>0$ independent of $x, r, \sigma, \varepsilon$. Hence the assertion of the lemma holds if we take $r_{0} \in\left(0, r^{\prime}\right]$ satisfying $r_{0}^{-\left(\delta-\delta_{0}\right) / \omega} \geq C^{\prime}$.

Proposition 3.2. Assume that $\Phi(x, t)$ satisfies ( $\Phi 5)$. If $0 \leq \varepsilon_{1} \leq \varepsilon_{2} \leq \varepsilon_{0}$, $-\delta_{0} \leq \sigma_{j} \leq \sigma_{0}, j=1,2$ and

$$
\sigma_{1}+\frac{\delta-\delta_{0}}{\omega} \varepsilon_{1} \leq \sigma_{2}+\frac{\delta-\delta_{0}}{\omega} \varepsilon_{2}
$$

then $L^{\Phi_{\varepsilon_{1}}, \kappa_{\sigma_{1}}}(G) \subset L^{\Phi_{\varepsilon_{2}}, \kappa_{\sigma_{2}}}(G)$ and

$$
\|f\|_{\Phi_{\varepsilon_{2}}, \kappa_{\sigma_{2}} ; G} \leq C\|f\|_{\Phi_{\varepsilon_{1}}, \kappa_{\sigma_{1}} ; G}
$$

for all $f \in L^{\Phi_{\varepsilon_{1}}, \kappa_{\sigma_{1}}}(G)$ with $C>0$ independent of $\varepsilon_{1}, \varepsilon_{2}, \sigma_{1}, \sigma_{2}$. In particular,

$$
L^{\Phi, \kappa}(G) \subset L^{\Phi_{\varepsilon}, \kappa_{\sigma}}(G)
$$

if $0 \leq \varepsilon \leq \varepsilon_{0},-\delta_{0} \leq \sigma \leq \sigma_{0}$ and $\sigma+\left(\left(\delta-\delta_{0}\right) / \omega\right) \varepsilon \geq 0$.

Proof. Let $\|f\|_{\Phi_{\varepsilon_{1}}, \kappa_{\sigma_{1}} ; G} \leq 1$. Then

$$
\frac{\kappa_{\sigma_{1}}(x, r)}{|B(x, r)|} \int_{B(x, r)} \Phi_{\varepsilon_{1}}(y,|f(y)|) d y \leq 1
$$

for $x \in G$ and $0<r<d_{G}$.

For $x \in G$ and $0<r<d_{G}$, let

$$
k(x, r)=\Phi_{\varepsilon_{1}}^{-1}\left(x, \kappa_{\sigma_{1}}(x, r)^{-1}\right)
$$

and

$$
I(x, r)=\int_{B(x, r)} \Phi_{\varepsilon_{2}}(y,|f(y)|) d y .
$$

We write $I(x, r)=I_{1}(x, r)+I_{2}(x, r)$, where

$$
I_{1}(x, r)=\int_{B(x, r) \cap\{y:|f(y)| \leq k(x, r)\}} \Phi_{\varepsilon_{2}}(y,|f(y)|) d y
$$

and

$$
I_{2}(x, r)=\int_{B(x, r) \cap\{y:|f(y)|>k(x, r)\}} \Phi_{\varepsilon_{2}}(y,|f(y)|) d y .
$$

If $|f(y)| \leq k(x, r)$, then

$$
\Phi_{\varepsilon_{2}}(y,|f(y)|) \leq A_{2} \Phi_{\varepsilon_{2}}(y, k(x, r))=A_{2} k(x, r)^{\varepsilon_{1}-\varepsilon_{2}} \Phi_{\varepsilon_{1}}(y, k(x, r)) .
$$

Let $r_{0} \in\left(0, \min \left(1, d_{G}\right)\right)$ be the number given in Lemma 3.1. Then, (3.2) implies

$$
k(x, r) \leq C \kappa_{\sigma_{1}}(x, r)^{-1} \leq C r^{-N}
$$


for $0<r \leq r_{0}$ with constants independent of $x, \sigma_{1}, \varepsilon_{1}$. Hence, by $(\Phi 5)$, there is a constant $B>0$ independent of $x, \sigma_{1}, \varepsilon_{1}$, such that

$$
\Phi_{\varepsilon_{1}}(y, k(x, r)) \leq B \Phi_{\varepsilon_{1}}(x, k(x, r))
$$

whenever $|x-y|<r \leq r_{0}$. Therefore,

$$
I_{1}(x, r) \leq C|B(x, r)| k(x, r)^{\varepsilon_{1}-\varepsilon_{2}} \Phi_{\varepsilon_{1}}(x, k(x, r))=C|B(x, r)| k(x, r)^{\varepsilon_{1}-\varepsilon_{2}} \kappa_{\sigma_{1}}(x, r)^{-1}
$$

for $0<r \leq r_{0}$.

On the other hand, if $|f(y)|>k(x, r)$, then

$$
\Phi_{\varepsilon_{2}}(y,|f(y)|)=|f(y)|^{\varepsilon_{1}-\varepsilon_{2}} \Phi_{\varepsilon_{1}}(y,|f(y)|) \leq k(x, r)^{\varepsilon_{1}-\varepsilon_{2}} \Phi_{\varepsilon_{1}}(y,|f(y)|),
$$

so that

$$
I_{2}(x, r) \leq k(x, r)^{\varepsilon_{1}-\varepsilon_{2}} \int_{B(x, r)} \Phi_{\varepsilon_{1}}(y,|f(y)|) d y \leq|B(x, r)| k(x, r)^{\varepsilon_{1}-\varepsilon_{2}} \kappa_{\sigma_{1}}(x, r)^{-1}
$$

for $0<r \leq r_{0}$.

Therefore,

which implies

$$
I(x, r) \leq C|B(x, r)| k(x, r)^{\varepsilon_{1}-\varepsilon_{2}} \kappa_{\sigma_{1}}(x, r)^{-1},
$$

$$
\frac{\kappa_{\sigma_{2}}(x, r)}{|B(x, r)|} \int_{B(x, r)} \Phi_{\varepsilon_{2}}(y,|f(y)|) d y \leq C r^{\sigma_{2}-\sigma_{1}} k(x, r)^{\varepsilon_{1}-\varepsilon_{2}}
$$

for $0<r \leq r_{0}$. Since

$$
k(x, r)^{-1} \leq C r^{\left(\delta-\delta_{0}\right) / \omega}
$$

and $\sigma_{2}-\sigma_{1}+\left(\left(\delta-\delta_{0}\right) / \omega\right)\left(\varepsilon_{2}-\varepsilon_{1}\right) \geq 0$ by assumption,

$$
\frac{\kappa_{\sigma_{2}}(x, r)}{|B(x, r)|} \int_{B(x, r)} \Phi_{\varepsilon_{2}}(y,|f(y)|) d y \leq C r^{\sigma_{2}-\sigma_{1}+\left(\left(\delta-\delta_{0}\right) / \omega\right)\left(\varepsilon_{2}-\varepsilon_{1}\right)} \leq C
$$

for $0<r \leq r_{0}$ with positive constants $C$ 's independent of $x, \sigma_{j}, \varepsilon_{j}(j=1,2)$.

In case $r_{0}<r<d_{G}$, we see

$$
\begin{aligned}
I(x, r) & \leq A_{2} \int_{B(x, r)} \Phi_{\varepsilon_{2}}(y, 1) d y+\int_{B(x, r)} \Phi_{\varepsilon_{1}}(y,|f(y)|) d y \\
& \leq A_{1} A_{2}|B(x, r)|+|B(x, r)| \kappa_{\sigma_{1}}(x, r)^{-1},
\end{aligned}
$$

so that

$$
\frac{\kappa_{\sigma_{2}}(x, r)}{|B(x, r)|} \int_{B(x, r)} \Phi_{\varepsilon_{2}}(y,|f(y)|) d y \leq A_{1} A_{2} \kappa_{\sigma_{2}}(x, r)+r^{\sigma_{2}-\sigma_{1}} \leq C
$$

with $C$ independent of $r, x, \sigma_{1} \sigma_{2}$.

Therefore, $\|f\|_{\Phi_{\varepsilon_{2}}, \kappa_{\sigma_{2}} ; G} \leq C$ with $C>0$ independent of $\varepsilon_{1}, \varepsilon_{2}, \sigma_{1}, \sigma_{2}$.

Let $\eta(\varepsilon)$ be an increasing positive function on $(0, \infty)$ such that $\eta(0+)=0$. Let $\xi(\varepsilon)$ be a function on $\left(0, \varepsilon_{1}\right]$ with some $\varepsilon_{1} \in\left(0, \varepsilon_{0} / 2\right]$ such that $-\delta_{0} \leq \xi(\varepsilon) \leq \sigma_{0}$ for $0<\varepsilon \leq \varepsilon_{1}, \xi(0+)=0$ and $\varepsilon \mapsto \xi(\varepsilon)+\left(\left(\delta-\delta_{0}\right) / \omega\right) \varepsilon$ is non-decreasing; in particular, $\xi(\varepsilon)+\left(\left(\delta-\delta_{0}\right) / \omega\right) \varepsilon \geq 0$ for $0<\varepsilon \leq \varepsilon_{1}$.

Given $\Phi(x, t), \kappa(x, r), \eta(\varepsilon)$ and $\xi(\varepsilon)$, the associated (generalized) grand MusielakOrlicz-Morrey space is defined by (cf. [17] for generalized grand Morrey space)

$$
\widetilde{L}_{\eta, \xi}^{\Phi, \kappa}(G)=\left\{f \in \bigcap_{0<\varepsilon \leq \varepsilon_{1}} L^{\Phi_{\varepsilon}, \kappa_{\xi(\varepsilon)}}(G) ;\|f\|_{\Phi, \kappa ; \eta, \xi ; G}<\infty\right\},
$$


where

$$
\|f\|_{\Phi, \kappa ; \eta, \xi ; G}=\sup _{0<\varepsilon \leq \varepsilon_{1}} \eta(\varepsilon)\|f\|_{\Phi_{\varepsilon}, \kappa_{\xi(\varepsilon)} ; G} .
$$

$\widetilde{L}_{\eta, \xi}^{\Phi, \kappa}(G)$ is a Banach space with the norm $\|f\|_{\Phi, \kappa ; \eta, \xi ; G}$. Note that, in view of Proposition 3.2, this space is determined independent of the choice of $\varepsilon_{1}$.

In case $\xi(\varepsilon) \equiv 0$, the symbol $\xi$ may be omitted. If $\kappa(x, r)=r^{N}$ and $\xi(\varepsilon) \equiv 0$, then the symbol $\kappa$ will be also omitted; namely

$$
\widetilde{L}_{\eta}^{\Phi}(G)=\left\{f \in \bigcap_{0<\varepsilon \leq \varepsilon_{0}} L^{\Phi_{\varepsilon}}(G) ;\|f\|_{\Phi ; \eta ; G}:=\sup _{0<\varepsilon \leq \varepsilon_{0}} \eta(\varepsilon)\|f\|_{\Phi_{\varepsilon} ; G}<\infty\right\} .
$$

This space may be called a grand Musielak-Orlicz space.

Remark 3.3. The grand Musielak-Orlicz space $\widetilde{L}_{\eta}^{\Phi}(G)$ include the following spaces:

- generalized grand Lebesgue spaces introduced in [4];

- grand Orlicz spaces introduced in [18] where $\Phi(x, t)=\Phi(t)$ satisfying

$$
\sup _{0<\varepsilon \leq \varepsilon_{0}} \eta(\varepsilon) \int_{1}^{\infty} t^{-N-\varepsilon} \Phi(t) \frac{d t}{t}<\infty
$$

(see also [8]).

The (generalized) grand Musielak-Orlicz-Morrey space $\widetilde{L}_{\eta, \xi}^{\Phi, \kappa}(G)$ include also the following spaces:

- grand Morrey spaces introduced in [21] where $\xi(\varepsilon) \equiv 0$;

- grand grand Morrey spaces introduced in [28] and generalized grand Morrey spaces introduced in [17] where $\xi(\varepsilon)$ is an increasing positive function on $(0, \infty)$.

\section{Boundedness of the maximal operator}

Hereafter, we shall always assume that $\Phi(x, t)$ satisfies $(\Phi 5)$. For a nonnegative $f \in L_{\mathrm{loc}}^{1}(G), x \in G, 0<r<d_{G}$ and $\varepsilon>0$, set

$$
I(f ; x, r):=\frac{1}{|B(x, r)|} \int_{B(x, r) \cap G} f(y) d y
$$

and

$$
J_{\varepsilon}(f ; x, r):=\frac{1}{|B(x, r)|} \int_{B(x, r) \cap G} \Phi_{\varepsilon}(y, f(y)) d y .
$$

We show a Jensen type inequality for functions in $L^{\Phi_{\varepsilon}, \kappa_{\sigma}}(G)$.

Lemma 4.1. There exists a constant $C>0$ (independent of $\varepsilon$ and $\sigma$ ) such that

$$
\Phi_{\varepsilon}(x, I(f ; x, r)) \leq C J_{\varepsilon}(f ; x, r)
$$

for all $x \in G, 0<r<d_{G}, 0<\varepsilon \leq \varepsilon_{0}$ and for all nonnegative $f \in L_{\text {loc }}^{1}(G)$ such that $f(y) \geq 1$ or $f(y)=0$ for each $y \in G$ and $\|f\|_{\Phi_{\varepsilon}, \kappa_{\sigma} ; G} \leq 1$ with $-\delta_{0} \leq \sigma \leq \sigma_{0}$.

Proof. Let $f$ be as in the statement of the lemma and let $I=I(f ; x, r)$ and $J_{\varepsilon}=J_{\varepsilon}(f ; x, r)$ for $x \in G, 0<r<d_{G}$ and $0<\varepsilon \leq \varepsilon_{0}$. Note that $\|f\|_{\Phi_{\varepsilon}, \kappa_{\sigma} ; G} \leq 1$ implies $J_{\varepsilon} \leq 2 A_{3} \kappa_{\sigma}(x, r)^{-1}$ by $(3.1)$. 
By $(\Phi 2)$ and $(3.2), \Phi_{\varepsilon}(y, f(y)) \geq\left(A_{1} A_{2}\right)^{-1} f(y)$, since $f(y) \geq 1$ or $f(y)=0$. Hence $I \leq A_{1} A_{2} J_{\varepsilon}$. Thus, if $J_{\varepsilon} \leq 1$, then

$$
\Phi_{\varepsilon}(x, I) \leq\left(A_{1} A_{2} J_{\varepsilon}\right) A_{2} \phi\left(x, A_{1} A_{2}\right) \leq C J_{\varepsilon} .
$$

Next, suppose $J_{\varepsilon}>1$. Since $\Phi_{\varepsilon}(x, t) \rightarrow \infty$ as $t \rightarrow \infty$, there exists $K_{\varepsilon} \geq 1$ such that

$$
\Phi_{\varepsilon}\left(x, K_{\varepsilon}\right)=\Phi_{\varepsilon}(x, 1) J_{\varepsilon} .
$$

Then $K_{\varepsilon} \leq A_{2} J_{\varepsilon}$ by (3.2). With this $K_{\varepsilon}$, we have

$$
\int_{B(x, r) \cap G} f(y) d y \leq K_{\varepsilon}|B(x, r)|+A_{2} \int_{B(x, r) \cap G} f(y) \frac{f(y)^{-\varepsilon} \phi(y, f(y))}{K_{\varepsilon}^{-\varepsilon} \phi\left(y, K_{\varepsilon}\right)} d y .
$$

Since $\kappa_{\sigma}(x, r) J_{\varepsilon} \leq 2 A_{3}$,

$$
1 \leq K_{\varepsilon} \leq A_{2} J_{\varepsilon} \leq 2 A_{2} A_{3} \kappa_{\sigma}(x, r)^{-1} \leq C r^{-N}
$$

with a constant $C>0$ independent of $\varepsilon$ and $\sigma$. Hence, by $(\Phi 5)$ there is $\beta \geq 1$, independent of $f, x, r, \varepsilon$ and $\sigma$ such that

$$
\phi\left(x, K_{\varepsilon}\right) \leq \beta \phi\left(y, K_{\varepsilon}\right)
$$

for all $y \in B(x, r)$. Thus, we have

$$
\begin{aligned}
\int_{B(x, r) \cap G} f(y) d y & \leq K_{\varepsilon}|B(x, r)|+\frac{A_{2} \beta}{K_{\varepsilon}^{-\varepsilon} \phi\left(x, K_{\varepsilon}\right)} \int_{B(x, r) \cap G} \Phi_{\varepsilon}(y, f(y)) d y \\
& =K_{\varepsilon}|B(x, r)|+A_{2} \beta|B(x, r)| \frac{J_{\varepsilon}}{K_{\varepsilon}^{-\varepsilon} \phi\left(x, K_{\varepsilon}\right)} .
\end{aligned}
$$

Since

$$
K_{\varepsilon}^{-\varepsilon} \phi\left(x, K_{\varepsilon}\right)=K_{\varepsilon}^{-1} \Phi_{\varepsilon}\left(x, K_{\varepsilon}\right)=K_{\varepsilon}^{-1} J_{\varepsilon} \Phi_{\varepsilon}(x, 1) \geq A_{1}^{-1} K_{\varepsilon}^{-1} J_{\varepsilon},
$$

it follows that

so that by $(\Phi 2),(\Phi 3)$ and $(\Phi 4)$

$$
I \leq\left(1+A_{1} A_{2} \beta\right) K_{\varepsilon},
$$

$$
\Phi_{\varepsilon}(x, I) \leq C \Phi_{\varepsilon}\left(x, K_{\varepsilon}\right) \leq C J_{\varepsilon}
$$

with constants $C>0$ independent of $f, x, r, \varepsilon$ and $\sigma$ as required.

For a locally integrable function $f$ on $G$, the Hardy-Littlewood maximal function $M f$ is defined by

$$
M f(x)=\sup _{r>0} \frac{1}{|B(x, r)|} \int_{B(x, r) \cap G}|f(y)| d y .
$$

The following lemma can be proved in a way similar to the proof of [25, Theorem 1]:

Lemma 4.2. Let $p_{0}>1$ and $-\delta_{0} \leq \sigma \leq \sigma_{0}$. Then there exists a constant $C>0$ independent of $\sigma$ for which the following holds: If $f$ is a measurable function such that

$$
\int_{B(x, r) \cap G}|f(y)|^{p_{0}} d y \leq|B(x, r)| \kappa_{\sigma}(x, r)^{-1}
$$

for all $x \in G$ and $0<r<d_{G}$, then

$$
\int_{B(x, r) \cap G}[M f(y)]^{p_{0}} d y \leq C|B(x, r)| \kappa_{\sigma}(x, r)^{-1}
$$


for all $x \in G$ and $0<r<d_{G}$.

Lemma 4.3. There is a constant $C>0$ (independent of $\varepsilon$ and $\sigma$ ) such that

$$
\|M f\|_{\Phi_{\varepsilon}, \kappa_{\sigma} ; G} \leq C\|f\|_{\Phi_{\varepsilon}, \kappa_{\sigma} ; G}
$$

for all $f \in L^{\Phi_{\varepsilon}, \kappa_{\sigma}}(G)$ whenever $0<\varepsilon \leq \varepsilon_{0} / 2$ and $-\delta_{0} \leq \sigma \leq \sigma_{0}$.

Proof. Set $p_{0}=1+\varepsilon_{0} / 2$ and consider the function

$$
\tilde{\Phi}(x, t)=\Phi(x, t)^{1 / p_{0}} .
$$

Then $\tilde{\Phi}(x, t)$ also satisfies all the conditions $(\Phi j), j=1,2, \ldots, 5$ with $\varepsilon_{0}$ replaced by $\varepsilon_{0}^{\prime}=\varepsilon_{0} /\left(2+\varepsilon_{0}\right)$. In fact, it trivially satisfies $(\Phi j)$ for $j=1,2,4,5$. Since

$$
t^{-\varepsilon_{0}^{\prime}} t^{-1} \tilde{\Phi}(x, t)=\left[t^{-\varepsilon_{0}} \phi(x, t)\right]^{1 / p_{0}},
$$

condition ( $\Phi 3)$ implies that $\tilde{\Phi}(x, t)$ satisfies $(\Phi 3)$ with $\varepsilon_{0}$ replaced by $\varepsilon_{0}^{\prime}$.

Let $0<\varepsilon \leq \varepsilon_{0} / 2,-\delta_{0} \leq \sigma \leq \sigma_{0}$ and $f \geq 0$ and $\|f\|_{\Phi_{\varepsilon}, \kappa_{\sigma} ; G} \leq 1$. Let $f_{1}=$ $f \chi_{\{z: f(z) \geq 1\}}$ and $f_{2}=f-f_{1}$, where $\chi_{E}$ is the characteristic function of $E$.

Since $\Phi_{\varepsilon}(x, t) \geq 1 /\left(A_{1} A_{2}\right)$ for $t \geq 1$, we see that

$$
\tilde{\Phi}_{\varepsilon / p_{0}}(x, t)=\Phi_{\varepsilon}(x, t)^{1 / p_{0}} \leq\left(A_{1} A_{2}\right)^{1-1 / p_{0}} \Phi_{\varepsilon}(x, t)
$$

if $t \geq 1$, so that

$$
\int_{B(x, r) \cap G} \tilde{\Phi}_{\varepsilon / p_{0}}\left(y, f_{1}(y)\right) d y \leq 2\left(A_{1} A_{2}\right)^{1-1 / p_{0}} A_{3}|B(x, r)| \kappa_{\sigma}(x, r)^{-1}
$$

for every $x \in G$ and $0<r<d_{G}$. Hence $\left\|f_{1}\right\|_{\tilde{\Phi}_{\varepsilon / p_{0}}, \kappa_{\sigma} ; G} \leq c_{0}$ with $c_{0}>0$ independent of $\varepsilon$ and $\sigma$.

Let $F_{\varepsilon}(x)=\Phi_{\varepsilon}(x, f(x))$. Then $\tilde{\Phi}_{\varepsilon / p_{0}}(x, f(x))=F_{\varepsilon}(x)^{1 / p_{0}}$. Applying Lemma 4.1 to $\tilde{\Phi}_{\varepsilon / p_{0}}$ and $f_{1} / c_{0}$, we have

$$
\Phi_{\varepsilon}\left(x, M f_{1}(x)\right)=\left[\tilde{\Phi}_{\varepsilon / p_{0}}\left(x, M f_{1}(x)\right)\right]^{p_{0}} \leq C\left[M\left(F_{\varepsilon}^{1 / p_{0}}\right)(x)\right]^{p_{0}} .
$$

On the other hand, since $M f_{2} \leq 1$, we have by $(\Phi 2)$ and ( $\left.\Phi 3\right)$

$$
\Phi_{\varepsilon}\left(x, M f_{2}(x)\right) \leq A_{1} A_{2} .
$$

Thus, we obtain

$$
\Phi_{\varepsilon}(x, M f(x)) \leq C\left\{\left[M\left(F_{\varepsilon}^{1 / p_{0}}\right)(x)\right]^{p_{0}}+1\right\}
$$

for $x \in G$ with a constant $C>0$ independent of $f$ and $\varepsilon$. Hence

$$
\int_{B(x, r) \cap G} \Phi_{\varepsilon}(y, M f(y)) d y \leq C\left\{\int_{B(x, r) \cap G}\left[M\left(F_{\varepsilon}^{1 / p_{0}}\right)(y)\right]^{p_{0}} d y+|B(x, r)|\right\}
$$

for $x \in G$ and $0<r<d_{G}$. Since $\|f\|_{\Phi_{\varepsilon}, \kappa_{\sigma} ; G} \leq 1$ and $\Phi_{\varepsilon}(y, f(y))=F_{\varepsilon}(y)=$ $\left(F_{\varepsilon}^{1 / p_{0}}(y)\right)^{p_{0}}$, Lemma 4.2 implies

$$
\int_{B(x, r) \cap G}\left[M\left(F_{\varepsilon}^{1 / p_{0}}\right)(y)\right]^{p_{0}} d y \leq C|B(x, r)| \kappa_{\sigma}(x, r)^{-1}
$$

with a constant $C>0$ independent of $x, r, \varepsilon, \sigma$. Hence,

$$
\int_{B(x, r) \cap G} \Phi_{\varepsilon}(y, M f(y)) d y \leq C|B(x, r)| \kappa_{\sigma}(x, r)^{-1},
$$


which shows

$$
\|M f\|_{\Phi_{\varepsilon}, \kappa_{\sigma} ; G} \leq C\|f\|_{\Phi_{\varepsilon}, \kappa_{\sigma} ; G}
$$

with a constant $C>0$ independent of $\varepsilon$ and $\sigma$.

From this lemma we obtain the boundedness of the maximal operator on $\widetilde{L}_{\eta, \xi}^{\Phi, \kappa}(G)$.

Theorem 4.4. The maximal operator $M$ is bounded from $\widetilde{L}_{\eta, \xi}^{\Phi, \kappa}(G)$ into itself; namely there exists a constant $C>0$ such that

$$
\|M f\|_{\Phi, \kappa ; \eta, \xi ; G} \leq C\|f\|_{\Phi, \kappa ; \eta, \xi ; G}
$$

for all $f \in \widetilde{L}_{\eta, \xi}^{\Phi, \kappa}(G)$.

Corollary 4.5. If $\Phi_{p(\cdot),\left\{q_{j}(\cdot)\right\}}(x, t)$ satisfies the conditions in Example 2.1, then the maximal operator $M$ is bounded from $\widetilde{L}_{\eta}^{\Phi_{p(\cdot),\left\{q_{j}(\cdot)\right\}}}(G)$ into itself.

\section{Sobolev type inequality}

Lemma 5.1. [19, Lemma 5.1] Let $F(x, t)$ be a positive function on $G \times(0, \infty)$ satisfying the following conditions:

(F1) $F(x, \cdot)$ is continuous on $(0, \infty)$ for each $x \in G$;

(F2) there exists a constant $K_{1} \geq 1$ such that

$$
K_{1}^{-1} \leq F(x, 1) \leq K_{1} \quad \text { for all } x \in G ;
$$

(F3) $t \mapsto t^{-\varepsilon^{\prime}} F(x, t)$ is uniformly almost increasing for $\varepsilon^{\prime}>0$; namely there exists a constant $K_{2} \geq 1$ such that

$$
t^{-\varepsilon^{\prime}} F(x, t) \leq K_{2} s^{-\varepsilon^{\prime}} F(x, s) \text { for all } x \in G \text { whenever } 0<t<s .
$$

Set

$$
F^{-1}(x, s)=\sup \{t>0 ; F(x, t)<s\}
$$

for $x \in G$ and $s>0$. Then:

(1) $F^{-1}(x, \cdot)$ is non-decreasing;

(2) $F^{-1}(x, \lambda s) \leq\left(K_{2} \lambda\right)^{1 / \varepsilon^{\prime}} F^{-1}(x, s)$ for all $x \in G, s>0$ and $\lambda \geq 1$;

(3) $F\left(x, F^{-1}(x, t)\right)=t$ for all $x \in G$ and $t>0$;

(4) $K_{2}^{-1 / \varepsilon^{\prime}} t \leq F^{-1}(x, F(x, t)) \leq K_{2}^{2 / \varepsilon^{\prime}} t$ for all $x \in G$ and $t>0$;

(5) $\min \left\{1,\left(\frac{s}{K_{1} K_{2}}\right)^{1 / \varepsilon^{\prime}}\right\} \leq F^{-1}(x, s) \leq \max \left\{1,\left(K_{1} K_{2} s\right)^{1 / \varepsilon^{\prime}}\right\}$ for all $x \in G$ and $s>0$.

Remark 5.2. $F(x, t)=\Phi_{\varepsilon}(x, t)\left(0<\varepsilon \leq \varepsilon_{0}\right)$ satisfies (F1), (F2) and (F3) with $\varepsilon^{\prime}=1, K_{1}=A_{1}$ and $K_{2}=A_{2}$ and $F(x, t)=\kappa_{\sigma}(x, t)\left(-\delta_{0} \leq \sigma \leq \sigma_{0}\right)$ satisfies (F1), (F2) and (F3) with $\varepsilon^{\prime}=\delta-\delta_{0}, K_{1}=Q_{1}$ and $K_{2}=Q_{2}$.

Lemma 5.3. There exists a constant $C>0$ such that

$$
\frac{\eta(\varepsilon)}{|B(x, r)|} \int_{B(x, r) \cap G} f(y) d y \leq C \Phi_{\varepsilon}^{-1}\left(x, \kappa_{\xi(\varepsilon)}(x, r)^{-1}\right)
$$

for all $x \in G, 0<r<d_{G}, 0<\varepsilon \leq \varepsilon_{1}$ and nonnegative functions $f$ on $G$ such that $\|f\|_{\Phi, \kappa ; \eta, \xi ; G} \leq 1$. 
Proof. Let $f$ be a nonnegative function on $G$ such that $\|f\|_{\Phi, \kappa ; \eta, \xi ; G} \leq 1$. Then we have by (3.1)

$$
\frac{\kappa_{\xi(\varepsilon)}(x, r)}{|B(x, r)|} \int_{B(x, r) \cap G} \Phi_{\varepsilon}(y, \eta(\varepsilon) f(y)) d y \leq 2 A_{3}
$$

for all $x \in G, 0<r<d_{G}$ and $0<\varepsilon \leq \varepsilon_{1}$. Fix $\varepsilon$ and let $f_{1}=f \chi_{\{x: \eta(\varepsilon) f(x) \geq 1\}}$ and $f_{2}=f-f_{1}$. By Lemma 4.1,

$$
\Phi_{\varepsilon}\left(x, \frac{1}{|B(x, r)|} \int_{B(x, r) \cap G} \eta(\varepsilon) f_{1}(y) d y\right) \leq C \kappa_{\xi(\varepsilon)}(x, r)^{-1}
$$

for all $x \in G, 0<r<d_{G}$ and $0<\varepsilon \leq \varepsilon_{0} / 2$ with a constant $C>0$ independent of $x$, $r, \varepsilon$. Since

$$
\Phi_{\varepsilon}\left(x, \frac{1}{|B(x, r)|} \int_{B(x, r) \cap G} \eta(\varepsilon) f_{2}(y) d y\right) \leq A_{2} \Phi_{\varepsilon}(x, 1) \leq A_{1} A_{2},
$$

we have

$$
\Phi_{\varepsilon}\left(x, \frac{1}{|B(x, r)|} \int_{B(x, r) \cap G} \eta(\varepsilon) f(y) d y\right) \leq C_{1} \kappa_{\xi(\varepsilon)}(x, r)^{-1}
$$

with a constant $C_{1} \geq 1$ independent of $x, r, \varepsilon$. Hence, we find by Lemma 5.1 with $F=\Phi_{\varepsilon}$ and $\varepsilon^{\prime}=1$

$$
\begin{aligned}
\frac{1}{|B(x, r)|} \int_{B(x, r) \cap G} \eta(\varepsilon) f(y) d y & \leq A_{2} \Phi_{\varepsilon}^{-1}\left(x, C_{1} \kappa_{\xi(\varepsilon)}(x, r)^{-1}\right) \\
& \leq C_{1} A_{2}^{2} \Phi_{\varepsilon}^{-1}\left(x, \kappa_{\xi(\varepsilon)}(x, r)^{-1}\right)
\end{aligned}
$$

as required.

As a potential kernel, we consider a function

$$
J(x, r): G \times\left(0, d_{G}\right) \rightarrow[0, \infty)
$$

satisfying the following conditions:

(J1) $J(\cdot, r)$ is measurable on $G$ for each $r \in\left(0, d_{G}\right)$;

(J2) $J(x, \cdot)$ is non-increasing on $\left(0, d_{G}\right)$ for each $x \in G$;

(J3) $\int_{0}^{d_{G}} J(x, r) r^{N-1} d r \leq J_{0}<\infty$ for every $x \in G$.

Example 5.4. Let $\alpha(\cdot)$ be a measurable function on $G$ such that

$$
0<\alpha^{-}:=\inf _{x \in G} \alpha(x) \leq \sup _{x \in G} \alpha(x)=: \alpha^{+}<N .
$$

Then, $J(x, r)=r^{\alpha(x)-N}$ satisfies (J1), (J2) and (J3).

For a nonnegative measurable function $f$ on $G$, its $J$-potential $J f$ is defined by

$$
J f(x)=\int_{G} J(x,|x-y|) f(y) d y \quad(x \in G) .
$$

Set

$$
\bar{J}(x, r)=\frac{N}{r^{N}} \int_{0}^{r} J(x, \rho) \rho^{N-1} d \rho
$$

for $x \in G$ and $0<r<d_{G}$. Then $J(x, r) \leq \bar{J}(x, r)$. Further, $\bar{J}(x, \cdot)$ is non-increasing and continuous on $\left(0, d_{G}\right)$ for each $x \in G$. Also, set

$$
Y_{J}(x, r)=r^{N} \bar{J}(x, r)
$$


for $x \in G$ and $0<r<d_{G}$.

We consider the following condition:

$(\Phi \kappa J)$ there exist constants $\delta^{\prime}>0$ and $A_{4} \geq 1$ such that

$$
s^{\delta^{\prime}} Y_{J}(x, s) \Phi_{\varepsilon}^{-1}\left(x, \kappa_{\sigma}(x, s)^{-1}\right) \leq A_{4} t^{\delta^{\prime}} Y_{J}(x, t) \Phi_{\varepsilon}^{-1}\left(x, \kappa_{\sigma}(x, t)^{-1}\right)
$$

for all $x \in G$ whenever $0<t<s<d_{G}, 0<\varepsilon \leq \varepsilon_{0} / 2,-\delta_{0} \leq \sigma \leq \sigma_{0}$ and $\sigma+\left(\left(\delta-\delta_{0}\right) / \omega\right) \varepsilon \geq 0$.

Lemma 5.5. Assume $(\Phi \kappa J)$. Then there exists a constant $C>0$ such that

$$
\int_{r}^{d_{G}} \rho^{N} \Phi_{\varepsilon}^{-1}\left(x, \kappa_{\sigma}(x, \rho)^{-1}\right) d(-\bar{J}(x, \cdot))(\rho) \leq C Y_{J}(x, r) \Phi_{\varepsilon}^{-1}\left(x, \kappa_{\sigma}(x, r)^{-1}\right)
$$

for all $x \in G, 0<r \leq d_{G} / 2,0<\varepsilon \leq \varepsilon_{0} / 2$ and $-\min \left(\delta_{0},\left(\left(\delta-\delta_{0}\right) / \omega\right) \varepsilon\right) \leq \sigma \leq \sigma_{0}$.

Proof. We follow the proof of [19, Lemma 6.2], noting that the constants are independent of $\varepsilon$ and $\sigma$.

Lemma 5.6. Assume $(\Phi \kappa J)$. Then there exists a constant $C>0$ such that

$$
\eta(\varepsilon) \int_{G \backslash B(x, r)} J(x,|x-y|) f(y) d y \leq C Y_{J}(x, r) \Phi_{\varepsilon}^{-1}\left(x, \kappa_{\xi(\varepsilon)}(x, r)^{-1}\right)
$$

for all $x \in G, 0<r \leq d_{G} / 2,0<\varepsilon \leq \varepsilon_{1}$ and $f \geq 0$ satisfying $\|f\|_{\Phi, \kappa ; \eta, \xi ; G} \leq 1$.

Proof. By the integration by parts, we have

$$
\begin{aligned}
& \int_{G \backslash B(x, r)} J(x,|x-y|) f(y) d y \\
& \leq J\left(x, d_{G}-0\right) \int_{G} f(y) d y+\int_{r}^{d_{G}}\left(\int_{B(x, \rho) \cap G} f(y) d y\right) d(-J(x, \cdot))(\rho),
\end{aligned}
$$

where $J\left(x, d_{G}-0\right)=\lim _{\rho \rightarrow d_{G}-0} J(x, \rho)$. Hence, by Lemma 5.3, we have

$$
\begin{aligned}
\eta(\varepsilon) \int_{G \backslash B(x, r)} J(x,|x-y|) f(y) d y & \leq C\left\{Y_{J}\left(x, d_{G}\right) \Phi_{\varepsilon}^{-1}\left(x, \kappa_{\xi(\varepsilon)}\left(x, d_{G}\right)^{-1}\right)\right. \\
& \left.+\int_{r}^{d_{G}}|B(x, \rho)| \Phi_{\varepsilon}^{-1}\left(x, \kappa_{\xi(\varepsilon)}(x, \rho)^{-1}\right) d(-J(x, \cdot))(\rho)\right\} .
\end{aligned}
$$

Hence by $(\Phi \kappa J)$ and the previous lemma we obtain the required result.

Lemma 5.7. Assume $(\Phi \kappa J)$. Then there exists a constant $C>0$ such that

$$
\eta(\varepsilon) J f(x) \leq C\left\{\eta(\varepsilon) M f(x) Y_{J}\left(x, \kappa_{\xi(\varepsilon)}^{-1}\left(x, \Phi_{\varepsilon}(x, \eta(\varepsilon) M f(x))^{-1}\right)\right)+1\right\}
$$

for all $x \in G, 0<\varepsilon \leq \varepsilon_{1}$ and $f \geq 0$ satisfying $\|f\|_{\Phi, \kappa ; \eta, \xi ; G} \leq 1$.

Proof. Let $f$ be a nonnegative function on $G$ such that $\|f\|_{\Phi, \kappa ; \eta, \xi ; G} \leq 1$. For $0<r \leq d_{G} / 2$, we write

$$
\begin{aligned}
J f(x) & =\int_{B(x, r) \cap G} J(x,|x-y|) f(y) d y+\int_{G \backslash B(x, r)} J(x,|x-y|) f(y) d y \\
& =J_{1}(x)+J_{2}(x) .
\end{aligned}
$$

First note that

$$
J_{1}(x) \leq C Y_{J}(x, r) M f(x)
$$


(see, e.g., [30, p. 63, (16)]). By Lemma 5.6, we have

$$
\eta(\varepsilon) J_{2}(x) \leq C Y_{J}(x, r) \Phi_{\varepsilon}^{-1}\left(x, \kappa_{\xi(\varepsilon)}(x, r)^{-1}\right) .
$$

Hence

$$
\eta(\varepsilon) J f(x) \leq C Y_{J}(x, r)\left\{\eta(\varepsilon) M f(x)+\Phi_{\varepsilon}^{-1}\left(x, \kappa_{\xi(\varepsilon)}(x, r)^{-1}\right)\right\}
$$

for $x \in G, 0<r \leq d_{G} / 2$ and $0<\varepsilon \leq \varepsilon_{1}$.

We consider two cases.

Case 1: $d_{G} / 2<\kappa_{\xi(\varepsilon)}^{-1}\left(x, \Phi_{\varepsilon}(x, \eta(\varepsilon) M f(x))^{-1}\right)$. In this case, let $r=d_{G} / 2$. Since

$$
\Phi_{\varepsilon}(x, \eta(\varepsilon) M f(x)) \leq Q_{2} \kappa_{\xi(\varepsilon)}\left(x, d_{G} / 2\right)^{-1} \leq Q_{2} Q_{3} \max \left(1,\left(d_{G} / 2\right)^{-N}\right),
$$

it follows that $\eta(\varepsilon) M f(x) \leq C_{1}$ with a constant $C_{1}>0$ independent of $x$ and $\varepsilon$. Also,

$$
\Phi_{\varepsilon}^{-1}\left(x, \kappa_{\xi(\varepsilon)}(x, r)^{-1}\right)=\Phi_{\varepsilon}^{-1}\left(x, \kappa_{\xi(\varepsilon)}\left(x, d_{G} / 2\right)^{-1}\right) \leq C_{2}
$$

with a constant $C_{2}>0$ independent of $x$ and $\varepsilon$. Hence, by (5.1) and (J3),

$$
\eta(\varepsilon) J f(x) \leq C
$$

with a constant $C>0$ independent of $x$ and $\varepsilon$.

Case 2: $d_{G} / 2 \geq \kappa_{\xi(\varepsilon)}^{-1}\left(x, \Phi_{\varepsilon}(x, \eta(\varepsilon) M f(x))^{-1}\right)$. In this case, take

$$
r=\kappa_{\xi(\varepsilon)}^{-1}\left(x, \Phi_{\varepsilon}(x, \eta(\varepsilon) M f(x))^{-1}\right) .
$$

Then $\kappa_{\xi(\varepsilon)}(x, r)^{-1}=\Phi_{\varepsilon}(x, \eta(\varepsilon) M f(x))$, so that by Lemma 5.1(4)

$$
\Phi_{\varepsilon}^{-1}\left(x, \kappa_{\xi(\varepsilon)}(x, r)^{-1}\right) \leq C \eta(\varepsilon) M f(x)
$$

with a constant $C>0$ independent of $x$ and $\varepsilon$. Hence, by (5.1)

$$
\begin{aligned}
\eta(\varepsilon) J f(x) & \leq C Y_{J}(x, r) \eta(\varepsilon) M f(x) \\
& =C \eta(\varepsilon) M f(x) Y_{J}\left(x, \kappa_{\xi(\varepsilon)}^{-1}\left(x, \Phi_{\varepsilon}(x, \eta(\varepsilon) M f(x))^{-1}\right)\right)
\end{aligned}
$$

with a constant $C>0$ independent of $x$ and $\varepsilon$.

The following theorem gives a Sobolev type inequality for potentials $J f$ of $f \in$ $\widetilde{L}_{\eta, \xi}^{\Phi, \kappa}(G)$. Example 5.9 below shows that this theorem includes known Sobolev type inequalities as special cases.

Theorem 5.8. Assume $(\Phi \kappa J)$. Suppose a function

$$
\Psi(x, t): G \times[0, \infty) \rightarrow[0, \infty)
$$

satisfies $(\Phi 1)-(\Phi 4)$ with $\varepsilon_{0}$ replaced by some $\varepsilon_{0}^{\prime}$ in $(\Phi 3)$ and

$(\Psi \Phi)$ there exist a constant $A^{\prime} \geq 1$ and a strictly increasing continuous function $\zeta(\varepsilon)$ on $\left[0, \varepsilon_{1}\right]$ such that $\zeta(0)=0, \varepsilon \mapsto \xi(\varepsilon)+\left(\left(\delta-\delta_{0}\right) / \omega^{*}\right) \zeta(\varepsilon)$ is non-decreasing with $\omega^{*}>1$ such that $\Psi(x, t) \leq C t^{\omega^{*}}$ for $t \geq 1$, and

$$
\Psi_{\zeta(\varepsilon)}\left(x, t Y_{J}\left(x, \kappa_{\xi(\varepsilon)}^{-1}\left(x, \Phi_{\varepsilon}(x, t)^{-1}\right)\right)\right) \leq A^{\prime} \Phi_{\varepsilon}(x, t)
$$

for all $x \in G, t \geq 1$ and $0<\varepsilon \leq \varepsilon_{1}$.

Then there exists a constant $C>0$ such that

$$
\|J f\|_{\Psi, \kappa ; \eta \circ \zeta^{-1, \xi \circ \zeta^{-1} ; G}} \leq C\|f\|_{\Phi, \kappa ; \eta, \xi ; G}
$$

for all $f \in \widetilde{L}_{\eta, \xi}^{\Phi, \kappa}(G)$. 
Proof. Let $f$ be a nonnegative function on $G$ such that $\|f\|_{\Phi, \kappa ; \eta, \xi ; G} \leq 1$. Choose $\varepsilon_{1}^{\prime} \in\left(0, \varepsilon_{1}\right]$ such that $\zeta\left(\varepsilon_{1}^{\prime}\right) \leq \varepsilon_{0}^{\prime}$. Let $x \in G, 0<r<d_{G}$ and $0<\varepsilon \leq \varepsilon_{1}^{\prime}$. By Lemma 5.7 and $(\Psi \Phi)$ we have

$$
\begin{aligned}
& \Psi_{\zeta(\varepsilon)}(x, \eta(\varepsilon) J f(x)) \\
& \leq C\left\{\Psi_{\zeta(\varepsilon)}\left(x, \eta(\varepsilon) M f(x) Y_{J}\left(x, \kappa_{\xi(\varepsilon)}^{-1}\left(x, \Phi_{\varepsilon}(x, \eta(\varepsilon) M f(x))^{-1}\right)\right)\right)+1\right\} \\
& \leq C\left\{\Phi_{\varepsilon}(x, \eta(\varepsilon) M f(x))+1\right\} .
\end{aligned}
$$

Note that $\|f\|_{\Phi, \kappa ; \eta, \xi ; G} \leq 1$ implies $\|M f\|_{\Phi, \kappa ; \eta, \xi ; G} \leq C$ by Theorem 4.4. Hence there is a constant $C_{1}^{\prime}>0$ such that

$$
\frac{\kappa_{\xi(\varepsilon)}(x, r)}{|B(x, r)|} \int_{B(x, r) \cap G} \Phi_{\varepsilon}(y, \eta(\varepsilon) M f(y)) d y \leq C_{1}^{\prime}
$$

for all $x \in G, 0<r<d_{G}$ and $0<\varepsilon \leq \varepsilon_{1}^{\prime}$. Therefore, there is another constant $C_{2}^{\prime}>0$ such that

$$
\frac{\kappa_{\xi(\varepsilon)}(x, r)}{|B(x, r)|} \int_{B(x, r) \cap G} \Psi_{\zeta(\varepsilon)}(y, \eta(\varepsilon) J f(y)) d y \leq C_{2}^{\prime}
$$

for all $x \in G, 0<r<d_{G}$ and $0<\varepsilon \leq \varepsilon_{1}^{\prime}$, so that

$$
\frac{\kappa_{\left(\xi \circ \zeta^{-1}\right)\left(\varepsilon^{\prime}\right)}(x, r)}{|B(x, r)|} \int_{B(x, r) \cap G} \Psi_{\varepsilon^{\prime}}\left(y,\left(\eta \circ \zeta^{-1}\right)\left(\varepsilon^{\prime}\right) J f(y)\right) d y \leq C_{2}^{\prime}
$$

for all $x \in G, 0<r<d_{G}$ and $0<\varepsilon^{\prime} \leq \zeta\left(\varepsilon_{1}^{\prime}\right)$, which implies the required result.

Example 5.9. Let $\Phi(x, t)=\Phi_{p(\cdot),\left\{q_{j}(\cdot)\right\}}(x, t)$ be as in Example 2.1, $\kappa(x, r)=$ $r^{\nu(x)}(\log (e+1 / r))^{\beta(x)}$ be as in Example 2.2 and $J(x, r)=r^{\alpha(x)-N}$ be as in Example 5.4.

Note that $\sigma_{0}=0$ if $\nu^{+}:=\sup _{x \in G} \nu(x)=N$ and $0<\sigma_{0}<N-\nu^{+}$if $\nu^{+}<N$. We may take $0<\delta_{0}<\delta<\nu^{-}$and $\omega>p^{+}$. Then,

$$
\sigma+\frac{\delta-\delta_{0}}{\omega} \varepsilon<\sigma+\frac{\nu^{-}}{p^{+}} \varepsilon \leq \sigma+\frac{\nu(x)}{p(x)} \varepsilon .
$$

Hence, if $\sigma+\left(\left(\delta-\delta_{0}\right) / \omega\right) \varepsilon \geq 0$, then

$$
\frac{\nu(x)+\sigma}{p(x)-\varepsilon} \geq \frac{\nu(x)}{p(x)}
$$

Since

$Y_{J}(x, r) \Phi_{\varepsilon}^{-1}\left(x, \kappa_{\sigma}(x, r)^{-1}\right) \sim r^{\alpha(x)-(\nu(x)+\sigma) /(p(x)-\varepsilon)}\left[Q(x, 1 / r)(\log (e+1 / r))^{\beta(x)}\right]^{-1 /(p(x)-\varepsilon)}$, where $Q(x, t)=\prod_{j=1}^{k}\left(L^{(j)}(t)\right)^{q_{j}(x)}$, we see that condition $(\Phi \kappa J)$ holds if

$$
\inf _{x \in G}\left(\frac{\nu(x)}{p(x)}-\alpha(x)\right)>0 .
$$

Set

$$
\Psi(x, t)=\left[\Phi_{p(\cdot),\left\{q_{j}(\cdot)\right\}}(x, t)\right]^{p^{*}(x) / p(x)}(\log (e+t))^{p^{*}(x) \alpha(x) \beta(x) / \nu(x)},
$$

where $1 / p^{*}(x)=1 / p(x)-\alpha(x) / \nu(x)$. We see

$t Y_{J}\left(x, \kappa_{\sigma}^{-1}\left(x, \Phi_{\varepsilon}(x, t)^{-1}\right)\right) \sim t^{p(x) / p_{\sigma}^{*}(x)+\varepsilon \alpha(x) /(\nu(x)+\sigma)}\left[Q(x, t)(\log (e+t))^{\beta(x)}\right]^{-\alpha(x) /(\nu(x)+\sigma)}$, 
where $1 / p_{\sigma}^{*}(x)=1 / p(x)-\alpha(x) /(\nu(x)+\sigma)$. Hence

$$
\begin{aligned}
\Psi & \left(x, t Y_{J}\left(x, \kappa_{\sigma}^{-1}\left(x, \Phi_{\varepsilon}(x, t)^{-1}\right)\right)\right) \\
\sim & t^{p(x) p^{*}(x) / p_{\sigma}^{*}(x)+\varepsilon p^{*}(x) \alpha(x) /(\nu(x)+\sigma)}\left[Q(x, t)(\log (e+t))^{\beta(x)}\right]^{-p^{*}(x) \alpha(x) /(\nu(x)+\sigma)} \\
& \cdot Q(x, t)^{p^{*}(x) / p(x)}(\log (e+t))^{p^{*}(x) \alpha(x) \beta(x) / \nu(x)} \\
= & \Phi_{\varepsilon}(x, t) t^{\sigma\left(p^{*}(x)-p(x)\right) /(\nu(x)+\sigma)+\varepsilon\left[p^{*}(x) \alpha(x) /(\nu(x)+\sigma)+1\right]} \\
& \cdot Q(x, t)^{\sigma\left(p^{*}(x)-p(x)\right) /[p(x)(\nu(x)+\sigma)]}(\log (e+t))^{\sigma p^{*}(x) \alpha(x) \beta(x) /[\nu(x)(\nu(x)+\sigma)]} .
\end{aligned}
$$

Here, note that $\xi(\varepsilon)+\left(\nu^{-} / p^{+}\right) \varepsilon \geq 0$ implies $\nu(x)+\xi(\varepsilon)>\nu(x) / 2$ if $0<\varepsilon \leq 1 / 2$. Let $0<\varepsilon \leq \min \left(1 / 2, \varepsilon_{1}\right)$. Let $\theta=\left(\delta-\delta_{0}\right) / \omega$. Since

$$
\begin{aligned}
& \frac{\xi(\varepsilon)}{\nu(x)+\xi(\varepsilon)} \leq \frac{\xi(\varepsilon)+\theta \varepsilon}{\nu(x)} \text { and } \frac{p^{*}(x) \alpha(x)}{\nu(x)+\xi(\varepsilon)}+1 \leq 2 \frac{p^{*}(x)}{p(x)}, \\
& \Psi\left(x, t Y_{J}\left(x, \kappa_{\xi(\varepsilon)}^{-1}\left(x, \Phi_{\varepsilon}(x, t)^{-1}\right)\right)\right) \\
& \lesssim \Phi_{\varepsilon}(x, t) t^{(\xi(\varepsilon)+\theta \varepsilon)\left(p^{*}(x)-p(x)\right) / \nu(x)+2 \varepsilon p^{*}(x) / p(x)}[\log (e+t)]^{m_{1}(\xi(\varepsilon)+\theta \varepsilon)}
\end{aligned}
$$

for $t \geq 1$ with a constant $m_{1} \geq 0$. In view of $(5.2)$, we also see that

$$
t Y_{J}\left(x, \kappa_{\xi(\varepsilon)}^{-1}\left(x, \Phi_{\varepsilon}(x, t)^{-1}\right)\right) \gtrsim t^{p(x) / p^{*}(x)}[\log (e+t)]^{-m_{2}}
$$

with a constant $m_{2} \geq 0$, which implies

$$
\begin{aligned}
& \Psi_{\zeta(\varepsilon)}\left(x, t Y_{J}\left(x, \kappa_{\xi(\varepsilon)}^{-1}\left(x, \Phi_{\varepsilon}(x, t)^{-1}\right)\right)\right) \\
& \lesssim \Phi_{\varepsilon}(x, t)\left\{t^{p(x) / p^{*}(x)}[\log (e+t)]^{-m_{2}}\right\}^{-\zeta(\varepsilon)} \\
& \quad \cdot t^{2 p^{*}(x) / p(x) \varepsilon}\left\{t^{\left(p^{*}(x)-p(x)\right) / \nu(x)}[\log (e+t)]^{m_{1}}\right\}^{(\xi(\varepsilon)+\theta \varepsilon)}
\end{aligned}
$$

for $t \geq 1$.

Now, let $\zeta(\varepsilon)=a \varepsilon+b(\xi(\varepsilon)+\theta \varepsilon)(a, b>0)$. If $a>2 \sup _{x \in G}\left(p^{*}(x) / p(x)\right)^{2}$, then

$$
\sup _{x \in G, t \geq 1}\left\{t^{p(x) / p^{*}(x)}[\log (e+t)]^{-m_{2}}\right\}^{-a} t^{2 p^{*}(x) / p(x)}<\infty
$$

and if $b>\sup _{x \in G} p^{*}(x)\left(p^{*}(x)-p(x)\right) /(p(x) \nu(x))$, then

$$
\sup _{x \in G, t \geq 1}\left\{t^{p(x) / p^{*}(x)}[\log (e+t)]^{-m_{2}}\right\}^{-b}\left\{t^{\left(p^{*}(x)-p(x)\right) / \nu(x)}[\log (e+t)]^{m_{1}}\right\}<\infty,
$$

so that $\Psi(x, t)$ satisfies condition $(\Psi \Phi)$ with $\zeta(\varepsilon)=a \varepsilon+b(\xi(\varepsilon)+\theta \varepsilon)(0<\varepsilon \leq$ $\left.\min \left(1 / 2, \varepsilon_{1}\right)\right)$.

\section{Trudinger type inequality}

In this section, we consider Trudinger type inequality on $\widetilde{L}_{\eta, \xi}^{\Phi, \kappa}(G)$.

Lemma 6.1. Let $t_{1}, t_{2}>0$. If

$$
\Phi\left(x, t_{1}\right) \leq K \Phi\left(x, t_{2}\right)
$$

for some $x \in G$ with $K \geq A_{2}^{-1}$, then $t_{1} \leq A_{2} K t_{2}$. 
Proof. Assume $t_{1}>A_{2} K t_{2}$. Note that $t_{1}>t_{2}$. Using (Ф3), we have

$$
\Phi\left(x, t_{1}\right)=t_{1} \phi\left(x, t_{1}\right)>K t_{2} \phi\left(x, t_{2}\right)=K \Phi\left(x, t_{2}\right),
$$

which contradicts the assumption.

In this section, we assume:

$(\Xi) \xi(\varepsilon) \leq a \varepsilon$ for $0<\varepsilon \leq \varepsilon_{1}$ with some $a \geq 0$.

Recall that $\xi(\varepsilon) \geq-\left(\left(\delta-\delta_{0}\right) / \omega\right) \varepsilon$ by assumption. Let

$$
\varepsilon(r)=(\log (e+1 / r))^{-1}
$$

for $r>0$ and let $r_{1} \in\left(0, \min \left(1, d_{G}\right)\right)$ be such that $\varepsilon(r) \leq \varepsilon_{1}$ for $0<r \leq r_{1}$.

Lemma 6.2. There exists a constant $C \geq 1$ such that

$$
C^{-1} \Phi^{-1}\left(x, \kappa(x, r)^{-1}\right) \leq \Phi_{\varepsilon(r)}^{-1}\left(x, \kappa_{\xi(\varepsilon(r))}(x, r)^{-1}\right) \leq C \Phi^{-1}\left(x, \kappa(x, r)^{-1}\right)
$$

for all $x \in G$ and $0<r \leq r_{1}$.

Proof. Fix $x \in G$ and set

$$
t_{0}(r)=\Phi^{-1}\left(x, \kappa(x, r)^{-1}\right) \quad \text { and } \quad t(r)=\Phi_{\varepsilon(r)}^{-1}\left(x, \kappa_{\xi(\varepsilon(r))}(x, r)^{-1}\right)
$$

for $0<r \leq r_{1}$. Then

$$
\begin{aligned}
\Phi\left(x, t_{0}(r)\right) & =\kappa(x, r)^{-1}=r^{\xi(\varepsilon(r))} \kappa_{\xi(\varepsilon(r))}(x, r)^{-1} \\
& =r^{\xi(\varepsilon(r))} \Phi_{\varepsilon(r)}(x, t(r))=r^{\xi(\varepsilon(r))} t(r)^{-\varepsilon(r)} \Phi(x, t(r)) .
\end{aligned}
$$

Thus, in view of Lemma 6.1, it is enough to show that there exists a constant $K \geq 1$ independent of $x$ such that

$$
K^{-1} \leq r^{\xi(\varepsilon(r))} t(r)^{-\varepsilon(r)} \leq K
$$

for all $0<r \leq r_{1}$.

Note that

$$
e^{-a} \leq r^{a \varepsilon(r)} \leq r^{\xi(\varepsilon(r))} \leq r^{-\left(\left(\delta-\delta_{0}\right) / \omega\right) \varepsilon(r)} \leq e^{\left(\delta-\delta_{0}\right) / \omega}
$$

for $0<r \leq r_{1}$ and that

$$
Q_{3}^{-1} \leq \kappa(x, r)^{-1} \leq Q_{3}\left(1+\frac{1}{r}\right)^{N}
$$

by $(\kappa 3)$.

If $t(r) \leq 1$, then by (6.1) and (6.3)

$$
\begin{aligned}
Q_{3}^{-1} & \leq \kappa(x, r)^{-1}=r^{\xi(\varepsilon(r))} t(r)^{-\varepsilon(r)} \Phi(x, t(r)) \\
& \leq e^{\left(\delta-\delta_{0}\right) / \omega} t(r)^{1-\varepsilon(r)} \phi(x, t(r)) \leq e^{\left(\delta-\delta_{0}\right) / \omega} A_{1} A_{2} t(r)^{1-\varepsilon\left(d_{G}\right)},
\end{aligned}
$$

so that $t(r) \geq C_{1}^{-1}$ with a constant $C_{1} \geq 1$ independent of $x$. Thus

$$
C_{1}^{-\varepsilon\left(d_{G}\right)} \leq t(r)^{\varepsilon(r)} \leq 1
$$

if $t(r) \leq 1$.

If $t(r) \geq 1$, then by (6.1) and (6.3) again

$$
Q_{3}\left(1+\frac{1}{r}\right)^{N} \geq \kappa(x, r)^{-1} \geq e^{-a} t(r)^{1-\varepsilon(r)} \phi(x, t(r)) \geq e^{-a}\left(A_{1} A_{2}\right)^{-1} t(r)^{1-\varepsilon\left(d_{G}\right)},
$$


so that $t(r) \leq C_{2}\left[(1+1 / r)^{N}\right]^{1 /\left(1-\varepsilon\left(d_{G}\right)\right)}$ with $C_{2} \geq 1$ independent of $x$. Since $(1+$ $1 / r)^{\varepsilon(r)}$ is bounded for $r>0$, it follows that

$$
1 \leq t(r)^{\varepsilon(r)} \leq C_{2}^{\varepsilon\left(d_{G}\right)}\left[\left(1+\frac{1}{r}\right)^{N}\right]^{\varepsilon(r) /\left(1-\varepsilon\left(d_{G}\right)\right)} \leq C_{3}
$$

if $t(r) \geq 1$, with a constant $C_{3} \geq 1$ independent of $x$.

Therefore, (6.2) holds with $K=\max \left\{e^{\left(\delta-\delta_{0}\right) / \omega} C_{1}^{\varepsilon\left(d_{G}\right)}, e^{a} C_{3}\right\}$.

Lemma 6.3. There exists a constant $C>0$ such that

$$
\frac{1}{|B(x, r)|} \int_{B(x, r) \cap G} f(y) d y \leq C \Phi^{-1}\left(x, \kappa(x, r)^{-1}\right) \eta\left((\log (e+1 / r))^{-1}\right)^{-1}
$$

for all $x \in G, 0<r<d_{G}$ and nonnegative $f \in \widetilde{L}_{\eta, \xi}^{\Phi, \kappa}(G)$ with $\|f\|_{\Phi, \kappa ; \eta, \xi ; G} \leq 1$.

Proof. Let $f$ be a nonnegative measurable function on $G$ such that $\|f\|_{\Phi, \kappa ; \eta, \xi ; G} \leq$ 1.

If $0<r \leq r_{1}$, then by Lemma 5.3

$$
\frac{1}{|B(x, r)|} \int_{B(x, r) \cap G} f(y) d y \leq C \Phi_{\varepsilon(r)}^{-1}\left(x, \kappa_{\xi(\varepsilon(r))}(x, r)^{-1}\right) \eta(\varepsilon(r))^{-1}
$$

for all $x \in G$. Hence, using the above lemma we obtain (6.4).

In case $r_{1}<r<d_{G}$, note that

$$
\Phi_{\varepsilon\left(r_{1}\right)}^{-1}\left(x, \kappa_{\xi\left(\varepsilon\left(r_{1}\right)\right)}\left(x, r_{1}\right)^{-1}\right) \leq C \Phi^{-1}\left(x, \kappa(x, r)^{-1}\right)
$$

by $(\kappa 3)$ and Lemma $5.1(5)$. Hence, by Lemma 5.3 with $\varepsilon=\varepsilon\left(r_{1}\right)$, we obtain (6.4) in this case, too.

In this section, we also assume that

$\left(\mathrm{J}^{\prime}\right) J(x, r) \leq C_{J} r^{-\varsigma}$ for $x \in G$ and $0<r \leq d_{G}$ with constants $0 \leq \varsigma<N$ and $C_{J}>0$

(J4) there is $r_{0} \in\left(0, d_{G}\right)$ such that

$$
\inf _{x \in G} J\left(x, r_{0}\right)>0 \quad \text { and } \quad \inf _{x \in G} \frac{\bar{J}\left(x, r_{0}\right)}{\bar{J}\left(x, d_{G}\right)}>1 .
$$

Here note that $\left(\mathrm{J} 3^{\prime}\right)$ implies (J3).

Example 6.4. Let $\alpha(\cdot)$ and $J(x, r)$ be as in Example 5.4. Then, $J(x, r)$ satisfies $\left(\mathrm{J} 3^{\prime}\right)$ and $(\mathrm{J} 4)$ (with $\left.\varsigma=N-\alpha^{-}\right)$. In particular, it satisfies $(\mathrm{J} 4)$ with any $r_{0} \in\left(0, d_{G}\right)$.

We consider the function

$\Gamma(x, s)=\left\{\begin{array}{l}\int_{1 / s}^{d_{G}} \rho^{N} \Phi^{-1}\left(x, \kappa(x, \rho)^{-1}\right) \eta\left((\log (e+1 / \rho))^{-1}\right)^{-1} d(-\bar{J}(x, \cdot))(\rho) \text { if } s \geq \frac{1}{r_{0}}, \\ \Gamma\left(x, 1 / r_{0}\right) r_{0} s \quad \text { if } 0 \leq s \leq 1 / r_{0}\end{array}\right.$

for every $x \in G$, where $r_{0}$ is the number given in $(\mathrm{J} 4) . \Gamma(x, \cdot)$ is strictly increasing and continuous for each $x \in G$.

Lemma 6.5. There exist positive constants $C^{\prime}$ and $C^{\prime \prime}$ such that

(a) $\Gamma(x, s) \leq C^{\prime} s^{\varsigma} \eta\left((\log (e+s))^{-1}\right)^{-1}$ for all $x \in G$ and $s \geq 1 / r_{0}$ with $\varsigma$ in condition $\left(\mathrm{J} 3^{\prime}\right)$; 
(b) $\Gamma\left(x, 1 / r_{0}\right) \geq C^{\prime \prime}>0$ for all $x \in G$.

Proof. First note from $(\kappa 3)$ and Lemma 5.1(5) that

$$
C^{-1} \leq \Phi^{-1}\left(x, \kappa(x, r)^{-1}\right) \leq C r^{-N} .
$$

By (6.5) and $\left(\mathrm{J}^{\prime}\right)$,

$$
\begin{aligned}
\Gamma(x, s) & \leq C \eta\left((\log (e+s))^{-1}\right)^{-1} \int_{1 / s}^{d_{G}} d(-\bar{J}(x, \cdot))(\rho) \\
& \leq C \eta\left((\log (e+s))^{-1}\right)^{-1} \bar{J}(x, 1 / s) \leq C^{\prime} s^{\varsigma} \eta\left((\log (e+s))^{-1}\right)^{-1}
\end{aligned}
$$

for all $x \in G$ and $s \geq 1 / r_{0}$; and

$$
\begin{aligned}
\Gamma\left(x, 1 / r_{0}\right) & \geq C^{-1} \int_{r_{0}}^{d_{G}} \rho^{N} d(-\bar{J}(x, \cdot))(\rho) \geq C^{-1} r_{0}^{N} \int_{r_{0}}^{d_{G}} d(-\bar{J}(x, \cdot))(\rho) \\
& =C^{-1} r_{0}^{N}\left(\bar{J}\left(x, r_{0}\right)-\bar{J}\left(x, d_{G}\right)\right) \geq C^{\prime \prime}>0
\end{aligned}
$$

where we used $(\mathrm{J} 4)$ to obtain the inequalities in the last line.

Lemma 6.6. There exists a constant $C>0$ such that

$$
\int_{G \backslash B(x, \delta)} J(x,|x-y|) f(y) d y \leq C \Gamma\left(x, \frac{1}{\delta}\right)
$$

for all $x \in G, 0<\delta \leq r_{0}$ and nonnegative $f \in \widetilde{L}_{\eta, \xi}^{\Phi, \kappa}(G)$ with $\|f\|_{\Phi, \kappa ; \eta, \xi ; G} \leq 1$.

Proof. By integration by parts, Lemma 6.3, (6.5), (J3') and Lemma 6.5(b), we have

$$
\begin{aligned}
\int_{G \backslash B(x, \delta)} J(x,|x-y|) f(y) d y \leq \int_{G \backslash B(x, \delta)} \bar{J}(x,|x-y|) f(y) d y \\
\leq C\left\{d_{G}^{N} \bar{J}\left(x, d_{G}\right) \Phi^{-1}\left(x, \kappa\left(x, d_{G}\right)^{-1}\right) \eta\left(\left(\log \left(e+1 / d_{G}\right)\right)^{-1}\right)^{-1}\right. \\
\left.\quad+\int_{\delta}^{d_{G}} \rho^{N} \Phi^{-1}\left(x, \kappa(x, \rho)^{-1}\right) \eta\left((\log (e+1 / \rho))^{-1}\right)^{-1} d(-\bar{J}(x, \cdot))(\rho)\right\} \\
\leq C\left\{\Gamma\left(x, 1 / r_{0}\right)+\Gamma(x, 1 / \delta)\right\} \leq C \Gamma(x, 1 / \delta) .
\end{aligned}
$$

Lemma 6.7. Let $0<\lambda<N$ and define

$$
I_{\lambda} f(x)=\int_{G}|x-y|^{\lambda-N} f(y) d y
$$

for a nonnegative measurable function $f$ on $G$ and

$$
\omega_{\lambda}(z, r)=\frac{1}{1+\int_{r}^{d_{G}} \rho^{\lambda} \Phi^{-1}\left(z, \kappa(z, \rho)^{-1}\right) \eta\left((\log (e+1 / \rho))^{-1}\right)^{-1} \frac{d \rho}{\rho}}
$$

for $z \in G$. Then there exists a constant $C_{I, \lambda}>0$ such that

$$
\frac{\omega_{\lambda}(z, r)}{|B(z, r)|} \int_{B(z, r) \cap G} I_{\lambda} f(x) d x \leq C_{I, \lambda}
$$

for all $z \in G, 0<r<d_{G}$ and nonnegative $f \in \widetilde{L}_{\eta, \xi}^{\Phi, \kappa}(G)$ with $\|f\|_{\Phi, \kappa ; \eta, \xi ; G} \leq 1$. 
Proof. Let $z \in G$. Let $f(x)=0$ for $x \in \mathbf{R}^{N} \backslash G$ and write

$$
\begin{aligned}
I_{\lambda} f(x) & =\int_{B(z, 2 r)}|x-y|^{\lambda-N} f(y) d y+\int_{G \backslash B(z, 2 r)}|x-y|^{\lambda-N} f(y) d y \\
& =I_{1}(x)+I_{2}(x)
\end{aligned}
$$

for $x \in G$. By Fubini's theorem,

$$
\begin{aligned}
\int_{B(z, r) \cap G} I_{1}(x) d x & =\int_{B(z, 2 r)}\left(\int_{B(z, r) \cap G}|x-y|^{\lambda-N} d x\right) f(y) d y \\
& \leq \int_{B(z, 2 r)}\left(\int_{B(y, 3 r)}|x-y|^{\lambda-N} d x\right) f(y) d y \\
& \leq C \int_{B(z, 2 r)}\left(\int_{0}^{3 r} t^{\lambda} \frac{d t}{t}\right) f(y) d y \leq \frac{C}{\lambda} r^{\lambda} \int_{B(z, 2 r)} f(y) d y .
\end{aligned}
$$

Now, by Lemma $6.3,(\kappa 2)$ and Lemma $5.1(2)$, we have

$$
\begin{aligned}
r^{\lambda} \int_{B(z, 2 r)} f(y) d y & \leq C r^{\lambda}|B(z, 2 r)| \Phi^{-1}\left(z, \kappa(z, 2 r)^{-1}\right) \eta\left((\log (e+1 /(2 r)))^{-1}\right)^{-1} \\
& \leq C|B(z, r)| \int_{r}^{2 r} \rho^{\lambda} \Phi^{-1}\left(z, \kappa(z, \rho)^{-1}\right) \eta\left((\log (e+1 / \rho))^{-1}\right)^{-1} \frac{d \rho}{\rho}
\end{aligned}
$$

if $0<r<d_{G} / 2$ and, by Lemma 6.3 and (6.5), we have

$$
\begin{aligned}
& r^{\lambda} \int_{B(z, 2 r)} f(y) d y=r^{\lambda} \int_{B\left(z, d_{G}\right)} f(y) d y \\
& \leq C d_{G}{ }^{\lambda}\left|B\left(z, d_{G}\right)\right| \Phi^{-1}\left(z, \kappa\left(z, d_{G}\right)^{-1}\right) \eta\left(\left(\log \left(e+1 / d_{G}\right)\right)^{-1}\right)^{-1} \leq C|B(z, r)|
\end{aligned}
$$

if $d_{G} / 2 \leq r<d_{G}$. Therefore

$$
\int_{B(z, r) \cap G} I_{1}(x) d x \leq \frac{C}{\lambda} \frac{|B(z, r)|}{\omega_{\lambda}(z, r)}
$$

for all $0<r<d_{G}$.

For $I_{2}$, first note that $I_{2}(x)=0$ if $x \in G$ and $r \geq d_{G} / 2$. Let $0<r<d_{G} / 2$. Since

$$
I_{2}(x) \leq C \int_{G \backslash B(z, 2 r)}|z-y|^{\lambda-N} f(y) d y \quad \text { for } \quad x \in B(z, r) \cap G,
$$

by integration by parts and Lemma 6.3 , we have

$$
\begin{aligned}
I_{2}(x) \leq & C\left\{d_{G}{ }^{\lambda} \Phi^{-1}\left(z, \kappa\left(z, d_{G}\right)^{-1}\right) \eta\left(\left(\log \left(e+1 / d_{G}\right)\right)^{-1}\right)^{-1}\right. \\
& \left.+\int_{2 r}^{d_{G}} \rho^{\lambda} \Phi^{-1}\left(z, \kappa(z, \rho)^{-1}\right) \eta\left((\log (e+1 / \rho))^{-1}\right)^{-1} \frac{d \rho}{\rho}\right\} \\
\leq & \frac{C}{\omega_{\lambda}(z, r)}
\end{aligned}
$$

for all $x \in B(z, r) \cap G$. Hence

$$
\int_{B(z, r) \cap G} I_{2}(x) d x \leq C \frac{|B(z, r)|}{\omega_{\lambda}(z, r)} .
$$

Thus this lemma is proved. 

tion:

From now on, we deal with the case $\Gamma(x, r)$ satisfies the uniform log-type condi-

$\left(\Gamma_{\log }\right)$ there exists a constant $c_{\Gamma}>0$ such that

$$
\Gamma\left(x, s^{2}\right) \leq c_{\Gamma} \Gamma(x, s)
$$

for all $x \in G$ and $s \geq 1$.

By $\left(\Gamma_{\log }\right)$, together with Lemma 6.5 , we see that $\Gamma(x, s)$ satisfies the uniform doubling condition in $s$ :

Lemma 6.8. [20, Lemma 4.2] For every $a>1$, there exists $b>0$ such that $\Gamma(x, a s) \leq b \Gamma(x, s)$ for all $x \in G$ and $s>0$.

Now we consider the following condition (J5):

(J5) there exists $0<\lambda<N-\varsigma$ such that $r \mapsto r^{N-\lambda} J(x, r)$ is uniformly almost increasing on $\left(0, d_{G}\right)$ for $\varsigma$ in condition $\left(\mathrm{J}^{\prime}\right)$.

Example 6.9. Let $J$ be as in Example 5.4. It satisfies (J5) with $0<\lambda<\alpha^{-}$.

Theorem 6.10. Assume that $\Gamma$ satisfies $\left(\Gamma_{\log }\right)$ and $J$ satisfies $(J 5)$. For each $x \in G$, let $\gamma(x)=\sup _{s>0} \Gamma(x, s)$. Suppose $\Lambda(x, t): G \times[0, \infty) \rightarrow[0, \infty]$ satisfies the following conditions:

$(\Lambda 1) \Lambda(\cdot, t)$ is measurable on $G$ for each $t \in[0, \infty) ; \Lambda(x, \cdot)$ is continuous on $[0, \infty)$ for each $x \in G$;

$(\Lambda 2)$ there is a constant $A_{1}^{\prime} \geq 1$ such that $\Lambda(x, t) \leq \Lambda\left(x, A_{1}^{\prime} s\right)$ for all $x \in G$ whenever $0<t<s$;

$(\Lambda 3) \Lambda\left(x, \Gamma(x, s) / A_{2}^{\prime}\right) \leq A_{3}^{\prime} s$ for all $x \in G$ and $s>0$ with constants $A_{2}^{\prime}, A_{3}^{\prime} \geq 1$ independent of $x$.

Then, for $\lambda$ given in (J5), there exists a constant $C^{*}>0$ such that $J f(x) / C^{*} \leq \gamma(x)$ for a.e. $x \in G$ and

$$
\frac{\omega_{\lambda}(z, r)}{|B(z, r)|} \int_{B(z, r) \cap G} \Lambda\left(x, \frac{J f(x)}{C^{*}}\right) d x \leq 1
$$

for all $z \in G, 0<r<d_{G}$ and nonnegative $f \in \widetilde{L}_{\eta, \xi}^{\Phi, \kappa}(G)$ with $\|f\|_{\Phi, \kappa ; \eta, \xi ; G} \leq 1$.

By $\left(\Gamma_{\log }\right)$ and $(\Lambda 3)$, the assertion of this theorem can be considered as exponential integrability of $J f$; cf. Corollary 6.12 below.

Proof. Let $f$ be a nonnegative measurable function on $G$ such that $\|f\|_{\Phi, \kappa ; \eta, \xi ; G} \leq$ 1. Fix $x \in G$. For $0<\delta \leq r_{0}$, Lemma 6.6, (J5) and (J3') imply

$$
\begin{aligned}
J f(x) & \leq \int_{B(x, \delta)} J(x,|x-y|) f(y) d y+C \Gamma\left(x, \frac{1}{\delta}\right) \\
& =\int_{B(x, \delta)}|x-y|^{N-\lambda} J(x,|x-y|)|x-y|^{\lambda-N} f(y) d y+C \Gamma\left(x, \frac{1}{\delta}\right) \\
& \leq C\left\{\delta^{N-\lambda} J(x, \delta) I_{\lambda} f(x)+\Gamma\left(x, \frac{1}{\delta}\right)\right\} \\
& \leq C\left\{\delta^{N-\varsigma-\lambda} I_{\lambda} f(x)+\Gamma\left(x, \frac{1}{\delta}\right)\right\}
\end{aligned}
$$

with constants $C>0$ independent of $x$. 
If $I_{\lambda} f(x) \leq 1 / r_{0}$, then we take $\delta=r_{0}$. Then, by Lemma 6.5(b)

$$
J f(x) \leq C \Gamma\left(x, \frac{1}{r_{0}}\right) .
$$

By Lemma 6.8, there exists $C_{1}^{*}>0$ independent of $x$ such that

$$
J f(x) \leq C_{1}^{*} \Gamma\left(x, \frac{1}{2 A_{3}^{\prime}}\right) \quad \text { if } I_{\lambda} f(x) \leq 1 / r_{0} .
$$

Next, suppose $1 / r_{0}<I_{\lambda} f(x)<\infty$. Let $m=\sup _{s \geq 1 / r_{0}, x \in G} \Gamma(x, s) / s$. By $\left(\Gamma_{\log }\right)$, $m<\infty$. Define $\delta$ by

$$
\delta^{N-\varsigma-\lambda}=\frac{r_{0}^{N-\varsigma-\lambda}}{m} \Gamma\left(x, I_{\lambda} f(x)\right)\left(I_{\lambda} f(x)\right)^{-1} .
$$

Since $\Gamma\left(x, I_{\lambda} f(x)\right)\left(I_{\lambda} f(x)\right)^{-1} \leq m, 0<\delta \leq r_{0}$. Then by Lemma 6.5(b)

$$
\begin{aligned}
\frac{1}{\delta} & \leq C \Gamma\left(x, I_{\lambda} f(x)\right)^{-1 /(N-\varsigma-\lambda)}\left(I_{\lambda} f(x)\right)^{1 /(N-\varsigma-\lambda)} \\
& \leq C \Gamma\left(x, 1 / r_{0}\right)^{-1 /(N-\varsigma-\lambda)}\left(I_{\lambda} f(x)\right)^{1 /(N-\varsigma-\lambda)} \leq C\left(I_{\lambda} f(x)\right)^{1 /(N-\varsigma-\lambda)} .
\end{aligned}
$$

Hence, using $\left(\Gamma_{\log }\right)$ and Lemma 6.8 , we obtain

$$
\Gamma\left(x, \frac{1}{\delta}\right) \leq \Gamma\left(x, C\left(I_{\lambda} f(x)\right)^{1 /(N-\varsigma-\lambda)}\right) \leq C \Gamma\left(x, I_{\lambda} f(x)\right) .
$$

By Lemma 6.8 again, we see that there exists a constant $C_{2}^{*}>0$ independent of $x$ such that

$$
J f(x) \leq C_{2}^{*} \Gamma\left(x, \frac{1}{2 C_{I, \lambda} A_{3}^{\prime}} I_{\lambda} f(x)\right) \text { if } 1 / r_{0}<I_{\lambda} f(x)<\infty,
$$

where $C_{I, \lambda}$ is the constant given in Lemma 6.7.

Now, let $C^{*}=A_{1}^{\prime} A_{2}^{\prime} \max \left(C_{1}^{*}, C_{2}^{*}\right)$. Then, by (6.6) and (6.7),

$$
\frac{J f(x)}{C^{*}} \leq \frac{1}{A_{1}^{\prime} A_{2}^{\prime}} \max \left\{\Gamma\left(x, \frac{1}{2 A_{3}^{\prime}}\right), \Gamma\left(x, \frac{1}{2 C_{I, \lambda} A_{3}^{\prime}} I_{\lambda} f(x)\right)\right\}
$$

whenever $I_{\lambda} f(x)<\infty$. Since $I_{\lambda} f(x)<\infty$ for a.e. $x \in G$ by Lemma 6.7, $J f(x) / C^{*} \leq$ $\gamma(x)$ a.e. $x \in G$, and by $(\Lambda 2)$ and $(\Lambda 3)$, we have

$$
\begin{aligned}
\Lambda\left(x, \frac{J f(x)}{C^{*}}\right) & \leq \max \left\{\Lambda\left(x, \Gamma\left(x, \frac{1}{2 A_{3}^{\prime}}\right) / A_{2}^{\prime}\right), \Lambda\left(x, \Gamma\left(x, \frac{1}{2 C_{I, \lambda} A_{3}^{\prime}} I_{\lambda} f(x)\right) / A_{2}^{\prime}\right)\right\} \\
& \leq \frac{1}{2}+\frac{1}{2 C_{I, \lambda}} I_{\lambda} f(x)
\end{aligned}
$$

for a.e. $x \in G$. Thus, noting that $\omega_{\lambda}(z, r) \leq 1$ and using Lemma 6.7 , we have

$$
\begin{aligned}
& \frac{\omega_{\lambda}(z, r)}{|B(z, r)|} \int_{B(z, r) \cap G} \Lambda\left(x, \frac{J f(x)}{C^{*}}\right) d x \\
& \leq \frac{1}{2} \omega_{\lambda}(z, r)+\frac{1}{2 C_{I, \lambda}} \frac{\omega_{\lambda}(z, r)}{|B(z, r)|} \int_{B(z, r) \cap G} I_{\lambda} f(x) d x \leq \frac{1}{2}+\frac{1}{2}=1
\end{aligned}
$$

for all $z \in G$ and $0<r<d_{G}$. 
Remark 6.11. If $\Gamma(x, s)$ is bounded, that is,

$$
\sup _{x \in G} \int_{0}^{d_{G}} \rho^{N} \Phi^{-1}\left(x, \kappa(x, \rho)^{-1}\right) \eta\left((\log (e+1 / \rho))^{-1}\right)^{-1} d(-\bar{J}(x, \cdot))(\rho)<\infty,
$$

then by Lemma 6.6 we see that $J|f|$ is bounded for every $f \in \widetilde{L}_{\eta, \xi}^{\Phi, \kappa}(G)$. In particular, if $\omega_{N-\varsigma}(x, r)^{-1}$ is bounded, that is,

$$
\sup _{x \in G} \int_{0}^{d_{G}} \rho^{N-\varsigma} \Phi^{-1}\left(x, \kappa(x, \rho)^{-1}\right) \eta\left((\log (e+1 / \rho))^{-1}\right)^{-1} \frac{d \rho}{\rho}<\infty
$$

then $\Gamma(x, s)$ is bounded by $\left(\mathrm{J} 3^{\prime}\right)$, and hence $J|f|$ is bounded for every $f \in \widetilde{L}_{\eta, \xi}^{\Phi, \kappa}(G)$.

If we further assume a continuity of the potential kernel $J$ like condition $(\mathrm{J} 5)$ in our paper [20], then we can show a continuity of $J f$ for $f \in \widetilde{L}_{\eta, \xi}^{\Phi, \kappa}(G)$, as in [20, Theorem 5.3].

Applying Theorem 6.10 to special $\Phi, \kappa$ and $J$, we obtain the following corollary:

Corollary 6.12. Let $\kappa(x, r)$ and $\alpha(x)$ be as in Examples 2.2 and 5.4 and let $p(x)$ and $q(x)$ be as in Examples 2.1. Set $\eta(t)=t^{\theta}$ for $\theta>0, \Phi(x, t)=t^{p(x)}(\log (e+t))^{q(x)}$ and

$$
I_{\alpha(\cdot)} f(x)=\int_{G}|x-y|^{\alpha(x)-N} f(y) d y
$$

for a nonnegative locally integrable function $f$ on $G$. Assume that

$$
\alpha(x)-\nu(x) / p(x)=0 \quad \text { for all } x \in G .
$$

(1) Suppose that

$$
\inf _{x \in G}(-q(x) / p(x)-\beta(x) / p(x)+\theta+1)>0 .
$$

Then for $0<\lambda<\alpha^{-}$there exist constants $C^{*}>0$ and $C^{* *}>0$ such that $\frac{r^{\nu(z) / p(z)-\lambda}}{|B(z, r)|} \int_{B(z, r) \cap G} \exp \left(\left(\frac{I_{\alpha(\cdot)} f(x)}{C^{*}}\right)^{p(x) /(p(x)+\theta p(x)-\beta(x)-q(x))}\right) d x \leq C^{* *}$ (2) If

for all $z \in G, 0<r<d_{G}$ and nonnegative $f \in \widetilde{L}_{\eta, \xi}^{\Phi, \kappa}(G)$ with $\|f\|_{\Phi, \kappa ; \eta, \xi ; G} \leq 1$.

$$
\sup _{x \in G}(-q(x) / p(x)-\beta(x) / p(x)+\theta+1) \leq 0,
$$

then for $0<\lambda<\alpha^{-}$there exist constants $C^{*}>0$ and $C^{* *}>0$ such that

$$
\frac{r^{\nu(z) / p(z)-\lambda}}{|B(z, r)|} \int_{B(z, r) \cap G} \exp \left(\exp \left(\frac{I_{\alpha(\cdot)} f(x)}{C^{*}}\right)\right) d x \leq C^{* *}
$$

for all $z \in G, 0<r<d_{G}$ and nonnegative $f \in \widetilde{L}_{\eta, \xi}^{\Phi, \kappa}(G)$ with $\|f\|_{\Phi, \kappa ; \eta, \xi ; G} \leq 1$.

Proof. In the present situation, we see that

$$
\Gamma(x, s) \sim \begin{cases}(\log (e+s))^{-q(x) / p(x)-\beta(x) / p(x)+\theta+1} & \text { in case }(1), \\ \log (\log (e+s)) & \text { in case }(2)\end{cases}
$$

for all $x \in G$ and $s \geq 1 / r_{0}=2 / d_{G}$. Hence, we may take

$$
\Lambda(x, t)= \begin{cases}\exp \left(t^{p(x) /(p(x)+\theta p(x)-q(x)-\beta(x))}\right) & \text { in case }(1), \\ \exp (\exp t) & \text { in case }(2) .\end{cases}
$$


On the other hand,

$$
\omega_{\lambda^{\prime}}(z, r) \sim r^{\nu(z) / p(z)-\lambda^{\prime}}(\log (e+1 / r))^{-q(x) / p(x)-\beta(x) / p(x)+\theta}
$$

for all $z \in G, 0<s<d_{G}$ and $0<\lambda^{\prime}<\alpha^{-}$, so that

$$
r^{\nu(z) / p(z)-\lambda} \leq C \omega_{\lambda^{\prime}}(z, r)
$$

if $0<\lambda<\lambda^{\prime}<\alpha^{-}$. Thus, given $0<\lambda<\alpha^{-}$, Theorem 6.10 implies the required results.

\section{References}

[1] Adams, D. R.: A note on Riesz potentials. - Duke Math. J. 42, 1975, 765-778.

[2] Adams, D. R., and L. I. Hedberg: Function spaces and potential theory. - Springer, 1996.

[3] Almeida, A., J. Hasanov, and S. Samko: Maximal and potential operators in variable exponent Morrey spaces. - Georgian Math. J. 15, 2008, 195-208.

[4] Capone, C., M. R. Formica, and R. Giova: Grand Lebesgue spaces with respect to measurable functions. - Nonlinear Anal. 85, 2013, 125-131.

[5] Chiarenza, F., and M. Frasca: Morrey spaces and Hardy-Littlewood maximal function. Rend. Mat. Appl. (7) 7, 1987, 273-279.

[6] Cruz-Uribe, D., and A. Fiorenza: Variable Lebesgue spaces. Foundations and harmonic analysis. - Appl. Numer. Harmon. Anal., Birkhäuser/Springer, Heidelberg, 2013.

[7] Diening, L., P. Harjulehto, P. Hästö, and M. RŮŽIČKa: Lebesgue and Sobolev spaces with variable exponents. - Lecture Notes in Math. 2017, Springer, Heidelberg, 2011.

[8] Farroni, F., and R. Giova: The distance to $L^{\infty}$ in the grand Orlicz spaces. - J. Funct. Spaces Appl. 2013, Art. ID 658527, 1-7.

[9] Fiorenza, A., B. Gupta, and P. Jain: The maximal theorem in weighted grand Lebesgue spaces. - Studia Math. 188:2, 2008, 123-133.

[10] Fiorenza, A., and C. Sbordone: Existence and uniqueness results for solutions of nonlinear equations with right hand side in $L^{1}$. - Studia Math. 127:3, 1998, 223-231.

[11] Futamura, T., Y. Mizuta, and T. Ohno: Sobolev's theorem for Riesz potentials of functions in grand Morrey spaces of variable exponent. - In: Proceedings of the International Symposium on Banach and Function Spaces IV (Kitakyushu, Japan, 2012), 2014, 353-365.

[12] Greco, L., T. Iwaniec, and C. Sbordone: Inverting the $p$-harmonic operator. - Manuscripta Math. 92, 1997, 249-258.

[13] Guliyev, V.S., J. Hasanov, and S. Samko: Boundedness of the maximal, potential and singular operators in the generalized variable exponent Morrey spaces. - Math. Scand. 107, 2010, 285-304.

[14] Guliyev, V.S., J. Hasanov, and S. Samko: Boundedness of the maximal, potential and Singular integral operators in the generalized variable exponent Morrey type spaces. - J. Math. Sci. (N. Y.) 170:4, 2010, 423-443.

[15] Iwaniec, T., and C. Sbordone: On the integrability of the Jacobian under minimal hypotheses. - Arch. Ration. Mech. Anal. 119, 1992, 129-143.

[16] Iwaniec, T., and C. Sbordone: Riesz Transforms and elliptic pde's with VMO coefficients. - J. Anal. Math. 74, 1998, 183-212.

[17] Kokilashvili, V., A. Meskhi, and H. Rafeiro: Riesz type potential operators in generalized grand Morrey spaces. - Georgian Math. J. 20, 2013, 43-64.

[18] Koskela, P., and X. Zhong: Minimal assumptions for the integrability of the Jacobian. Ric. Mat. 51:2, 2002, 297-311. 
[19] Maeda, F.-Y., Y. Mizuta, T. Ohno, and T. Shimomura: Boundedness of maximal operators and Sobolev's inequality on Musielak-Orlicz-Morrey spaces. - Bull. Sci. Math. 137, 2013, 76-96.

[20] Maeda, F.-Y., Y. Mizuta, T. Ohno, and T. Shimomura: Trudinger's inequality and continuity of potentials on Musielak-Orlicz-Morrey spaces. - Potential Anal. 38, 2013, 515535.

[21] Meskhi, A.: Maximal functions, potentials and singular integrals in grand Morrey spaces. Complex Var. Elliptic Equ. 56:10-11, 2011, 1003-1019.

[22] Mizuta, Y., E. Nakai, T. Ohno and T. Shimomura: Riesz potentials and Sobolev embeddings on Morrey spaces of variable exponent. - Complex Var. Elliptic Equ. 56:7-9, 2011, 671-695.

[23] Mizuta, Y., and T. Shimomura: Sobolev embeddings for Riesz potentials of functions in Morrey spaces of variable exponent. - J. Math. Soc. Japan 60, 2008, 583-602.

[24] Morrey, C. B.: On the solutions of quasi-linear elliptic partial differential equations. - Trans. Amer. Math. Soc. 43, 1938, 126-166.

[25] NAKAI, E.: Hardy-Littlewood maximal operator, singular integral operators and the Riesz potentials on generalized Morrey spaces. - Math. Nachr. 166, 1994, 95-103.

[26] NAKAI, E.: Generalized fractional integrals on Orlicz-Morrey spaces. - In: Banach and function spaces (Kitakyushu, 2003), Yokohama Publ., Yokohama, 2004, 323-333.

[27] Peetre, J.: On the theory of $L_{p, \lambda}$ spaces. - J. Funct. Anal. 4, 1969, 71-87.

[28] Rafeiro, H.: A note on boundedness of operators in grand grand Morrey spaces. - In: Advances in harmonic analysis and operator theory, Oper. Theory Adv. Appl. 229, Birkhäuser/Springer Basel AG, Basel, 2013, 349-356.

[29] Sbordone, C.: Grand Sobolev spaces and their applications to variational problems. - Matematiche (Catania) 51:2, 1996, 335-347.

[30] Stein, E. M.: Singular integrals and differentiability properties of functions. - Princeton Univ. Press, Princeton, 1970.

Received 10 June $2014 \bullet$ Accepted 6 October 2014 